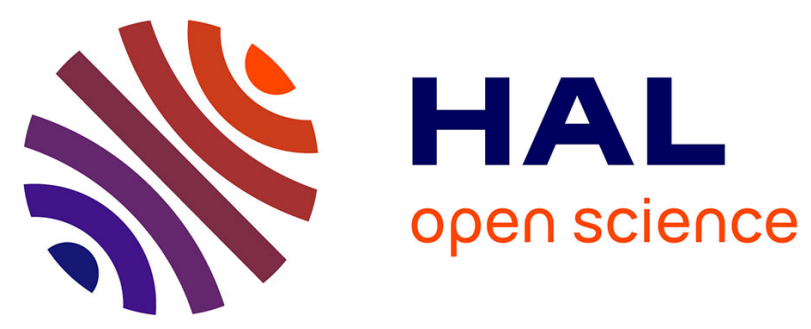

\title{
Quantum Dot Acceptors in Two-Dimensional Epitaxially Fused PbSe Quantum Dot Superlattices
}

Vincent Notot, Willem Walravens, Maxime Berthe, Nemanja Peric, Ahmed Addad, Xavier Wallart, Christophe Delerue, Zeger Hens, Bruno Grandidier, Louis Biadala

\section{To cite this version:}

Vincent Notot, Willem Walravens, Maxime Berthe, Nemanja Peric, Ahmed Addad, et al.. Quantum Dot Acceptors in Two-Dimensional Epitaxially Fused PbSe Quantum Dot Superlattices. ACS Nano, American Chemical Society, 2022, pp.acsnano.1c10596. 10.1021/acsnano.1c10596 . hal-03574889

\section{HAL Id: hal-03574889 \\ https://hal.archives-ouvertes.fr/hal-03574889}

Submitted on 15 Feb 2022

HAL is a multi-disciplinary open access archive for the deposit and dissemination of scientific research documents, whether they are published or not. The documents may come from teaching and research institutions in France or abroad, or from public or private research centers.
L'archive ouverte pluridisciplinaire HAL, est destinée au dépôt et à la diffusion de documents scientifiques de niveau recherche, publiés ou non, émanant des établissements d'enseignement et de recherche français ou étrangers, des laboratoires publics ou privés. 


\section{Quantum Dot Acceptors in Two-Dimensional}

\section{Epitaxially Fused PbSe Quantum Dot Superlattices}

Vincent Notot ${ }^{\dagger}$, Willem Walravens", Maxime Berthe ${ }^{\dagger}$, Nemanja Peric ${ }^{\dagger}$, Ahmed Addad ${ }^{\S}$, Xavier

Wallart $^{\dagger}$, Christophe Delerue ${ }^{\dagger}$,Zeger Hens', Bruno Grandidier ${ }^{\dagger, *}$ and Louis Biadala ${ }^{\dagger, *}$

${ }^{\dagger}$ Univ. Lille, CNRS, Centrale Lille, Univ. Polytechnique Hauts-de-France, JUNIA-ISEN, UMR 8520 - IEMN, F-59000 Lille, France.

§Univ. Lille, CNRS, INRAE, Centrale Lille, UMR 8207 - UMET - Unité Matériaux et Transformations, F-59000, Lille, France

" Physics and Chemistry of Nanostructures, Ghent University, 9000 Ghent, Belgium

\section{ABSTRACT}

Oriented attachment of colloidal quantum dots allows the growth of two-dimensional crystals by design, which could have striking electronic properties upon progress on manipulating their conductivity. Here, we explore the origin of doping in square and epitaxially fused $\mathrm{PbSe}$ quantum dot superlattices with low temperature scanning tunneling microscope and spectroscopy. Probing the density of states of numerous individual quantum dots reveals an electronic coupling between the hole ground states of the quantum dots. Moreover, a small amount of quantum dots shows a reproducible deep level in the band gap, which is not caused by structural defects in the 
connections but arises from unpassivated sites at the $\{111\}$ facets. Based on semiconductor statistics, these distinct defective quantum dots, randomly distributed in the superlattice, trap electrons, releasing a concentration of free holes, which is intimately related to the interdot electronic coupling. They act as acceptor quantum dots in the host quantum dot lattice, mimicking the role of dopant atoms in a semiconductor crystal.

KEYWORDS: Nanocrystal assemblies, quantum dot superlattice, electronic coupling, doping, defects, scanning tunneling microscopy 
Doping of semiconductor bulk materials is commonly achieved with the incorporation of foreign atoms in the crystals. Above a critical temperature, the counter charge carried by each dopant atom is released as a free charge carrier into the host lattice, which notably raises the semiconductor conductivity. Although numerous efforts have been devoted to improve the controlled substitution of host atoms with dopants over the years, obtaining successful electrically active substitutions turned out to be tricky in nanoscale materials. ${ }^{1-3}$ This is particularly true with colloidal semiconductor quantum dots (QDs), ${ }^{4}$ which have become ubiquitous in many applications based on thin films or QD solids. ${ }^{5,6}$ Albeit more complex than in bulk, the incorporation of aliovalent atoms acting as dopants in single QDs has been demonstrated, ${ }^{7,8}$ along with additional sources of doping due to the large surface-to-bulk ratio in QDs. The latter encompass doping from dangling bonds, surface impurities, remote charge transfer from the surrounding and deviation of stoichiometry in compounds. ${ }^{9-12}$ Concomitant to the quest for higher mobility in QD films, which can be achieved with ordered array of QDs and controlled interdot separation, ${ }^{13}$ maximizing the electronic coupling between QDs ${ }^{10,14,15}$ has offered alternative routes for electronic doping. For instance, the concept of substituting atoms in crystals was transposed to artificial atoms in QD solids by randomly introducing a small amount of metal nanocrystals in semiconductor QD superlattices. ${ }^{16,17}$ These nanocrystals act as individual dopants, significantly raising the superlattice conductivity.

With a growing interest in the formation of epitaxially connected QD superlattices, where a stronger electronic coupling between the QDs enhances the charge carrier mobility, ${ }^{18-21}$ doping faces distinct challenges. Indeed, the epitaxial connections of neighbor QDs involve ligand displacement and removal, ${ }^{22,23}$ at odds with the concept of remote doping, for which molecules adsorbed at the QD surface ensure an efficient charge transfer. Moreover, an increase of the 
conductivity in the superlattice requires an annealing up to $150^{\circ} \mathrm{C} .^{24}$ While such an annealing cures small structural defects, it might alter the electrical activity of remnant defects or impurities and might not leave $\mathrm{Au}$ or Ag nanocrystals unaltered when they are used as substitutional dopants. So far, the nature of the doping has been found to vary depending on the chemistry used to prepare epitaxially connected QD superlattices. ${ }^{24,25}$ But the microscopic nature of doping in these superlattices is still unknown. It is not clear if doping is caused by a charge transfer from the remaining ligands, which affects all the QDs in the superlattice or if doping is induced by isolated QDs which are defective and transfer extra free charge carriers to the superlattice.

Here, we investigate doping in two-dimensional (2D) epitaxially connected and annealed PbSe QD superlattices with scanning tunneling microscopy, which is a technique of choice to identify single dopants in semiconductors. ${ }^{26}$ The stripping of the lead oleate ligands from the $\{100\}$ facets by exposure to amines induces an epitaxial connection of the QDs, forming a $p$-type doped superlattice with a square geometry, where the unbound facets are still passivated with oleate ligands. ${ }^{24}$ As the knowledge of the electronic states for energies ranging between midgap to the band edges is essential to account for doping and transport, we first study the electronic structure of the superlattice at the local scale. Spatially-resolved tunneling spectroscopy shows that the occupied energy levels of a majority of QDs are affected by the neighboring QDs. This electronic coupling leads to the formation of extended valence band states, in analogy to semiconductor crystals described using band theory. Interestingly, within the superlattice, we find that a small amount of QDs exhibits in-gap states, which are caused by their surface chemistry and not by structural defects. These states are about $200 \mathrm{meV}$ above the valence band edge, their wave function being exceptionally delocalized over the whole QD. According to semiconductor statistics, the in-gap states can trap electrons, making the QDs negatively charged with a bound 
hole. Essential for the doping efficiency, notably at $77 \mathrm{~K}$, is the linewidth of the hole ground state resulting from the interdot electronic coupling, so that the defective QDs behave as genuine QD acceptors in epitaxially connected QD superlattice.

\section{RESULTS AND DISCUSSION}

In this study, we consider superlattices of epitaxially connected PbSe QDs (Figure 1a) having a mean diameter of $5.8 \mathrm{~nm}$ (dispersion 5\%). The superlattices were prepared according to the protocol described in ref. 24 and transferred either on a gold substrate to study their electronic properties with Scanning Tunneling Microscopy (STM) in ultrahigh vacuum or on carbon grids to unveil their structural properties with Scanning Transmission Electron Microscopy (STEM). Prior to their characterization, the samples were annealed between $120^{\circ} \mathrm{C}$ and $150^{\circ} \mathrm{C}$ for an hour in vacuum or ultrahigh vacuum (UHV). Figure 1c shows a STEM image of the annealed superlattice, where the QDs are arranged in a square lattice. The QDs are epitaxially connected by their ligand-

free (100) facets, leaving native ligands on the (111) facets, as illustrated in Figure 1b. Although a high degree of crystallinity exists at the QD-QD interface, a close examination of these interfaces in Figure 1c reveals that their area varies from QD to QD, giving rise to more or less wide pores between the attached QDs. We note that the annealing process does not increase the number of necks, keeping it at 3.0 \pm 0.05 per QD, does not change the surface chemistry if performed below $235^{\circ} \mathrm{C}$, but significantly improves the quality of the attachment between the QDs by reducing the defect observed in as-grown superlattices (Figure S1-S3), as found by Walravens et al. using high resolution TEM, X-ray fluorescence, X-ray diffraction, thermal gravimetric/differential thermal 
analyses. ${ }^{24}$ Moreover, this preparation step is mandatory to ensure stable and reproducible spectroscopic measurements with STM (Figure S4).

(a)

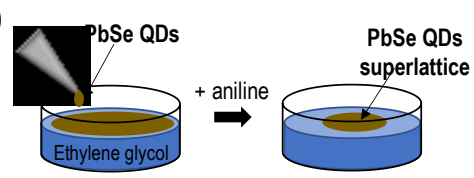

STEM

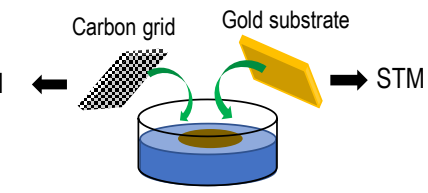

(b)

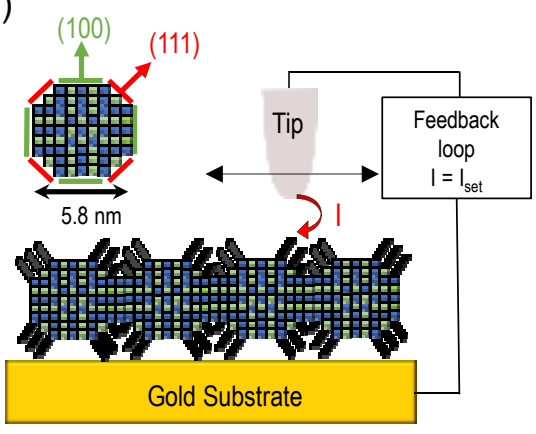

(c)

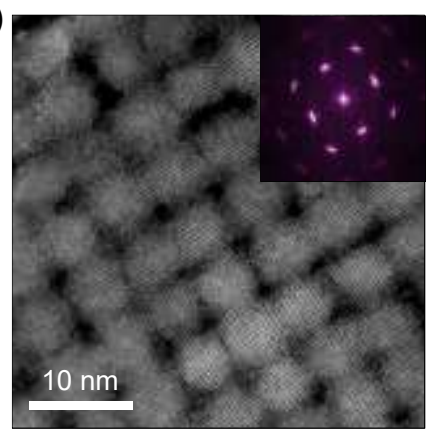

(d)

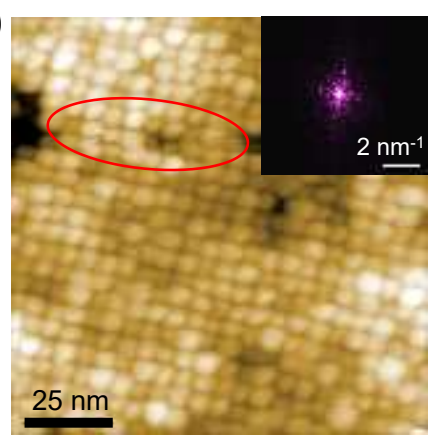

(e)

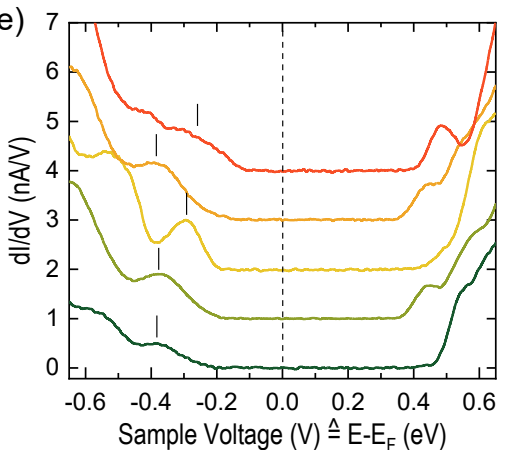

(f)

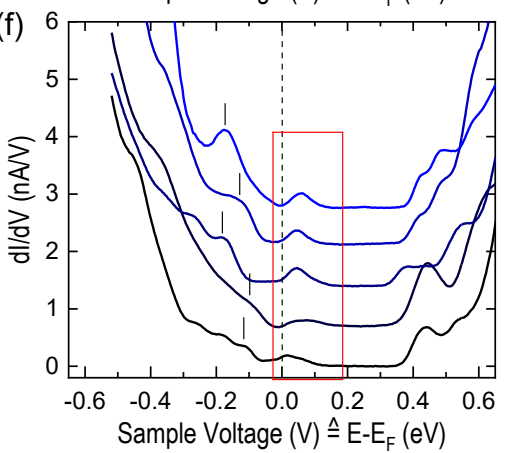

Figure 1. (a) Illustration of the superlattice synthesis and its transfer on various substrates. (b) Facet orientations of a PbSe QD and simplified schematic of the epitaxially connected PbSe QDs characterized with scanning tunneling microscopy. The $\{111\}$ facets are passivated with oleate ligands, which ensure the formation of double barrier tunnel junction between the STM tip and the gold substrate. (c) HAADF-STEM image of an annealed superlattice of PbSe QDs. Inset: FFT image showing the high crystallinity of the superlattice. (d) STM image of a superlattice showing the close-packing of PbSe QDs in a square superlattice $\left(\mathrm{V}_{\mathrm{S}}=+2.5 \mathrm{~V} ; \mathrm{I}_{\mathrm{set}}=50 \mathrm{pA}\right)$. The red oval encircles fuzzy QDs highlighting a strong interaction of the ligands with the STM tip. Inset: Corresponding Fast Fourier Transform Image. (e) Examples of tunneling spectra acquired on five different individual QDs, where a zero-conductance region is clearly measured on both sides of the zero bias $\left(\mathrm{V}_{\mathrm{S}}=+0.8 \mathrm{~V}\right.$; $I_{\text {set }}=200 \mathrm{pA}$ ). (f) Examples of tunneling spectra acquired on five different individual QDs showing in gap states at low positive bias, in the region delineated by the red rectangle $\left(\mathrm{V}_{\mathrm{S}}=+0.8 \mathrm{~V} ; \mathrm{I}_{\mathrm{set}}=200 \mathrm{pA}\right)$. 
The STM images are obtained in a constant current mode, for which the tunneling current is maintained by adjusting the height, $z$, of the tip with a feedback loop. A typical $100 \times 100 \mathrm{~nm}^{2}$ STM image of an annealed superlattice of PbSe QDs is shown in Figure 1d. Individual QDs arranged in a square lattice are clearly resolved. The long-range ordering of the lattice allows the observation of sharp spots in the Fast Fourier Transform (FFT) of the STM image. From the spot spacing, we determine a $5.8 \mathrm{~nm}$ periodicity for the lattice, yielding a much better agreement between the mean size of the QDs measured with STEM and STM than the one usually encountered for compact monolayers of QDs passivated with ligands. ${ }^{14,27}$ This correspondence shows that the QDs in the superlattice examined with STM are epitaxially connected and indicates that the size of the QDs can be directly measured from high-resolution STM images. It also suggests that the small fluctuations of the STM contrast in the region of connection between the QDs are caused by different widths of the crystalline necks, similar to what is observed with STEM. We note that a small number of QDs appears much brighter in the STM image of the superlattice, albeit featuring a diameter comparable to their QD neighbors. A closer inspection (Figure S5) reveals that this height difference measured in the topography can be explained by an asymmetric fusion of opposite facets between QD neighbors. Such a process leads to a vertical shift of the QD, corresponding to a buckled QD in the superlattice (Figure S5c), a feature that cannot be probed by STEM.

To examine the electronic properties of the QDs, we performed Scanning Tunneling Spectroscopy (STS) on individual QDs. A typical differential conductance spectrum measured on an isolated PbSe QD shows tunneling resonances on both sides of a zero-conductance region (ZCR). ${ }^{28,29}$ In the $\mathrm{ZCR}$, the Fermi level is measured at $0 \mathrm{~V}$ and is well separated from the 
resonances. A quick survey of spectra acquired on twelve different QDs randomly selected on the sample reveals two types of behavior: (1) spectra consistent with the existence of a ZCR (Figure 1e) and (2) spectra where a peak is observed just above the Fermi level at positive bias, filling a large part of the ZCR (Figure 1f). We start our analysis with the first set, which corresponds to most of the spectra measured in the superlattice.
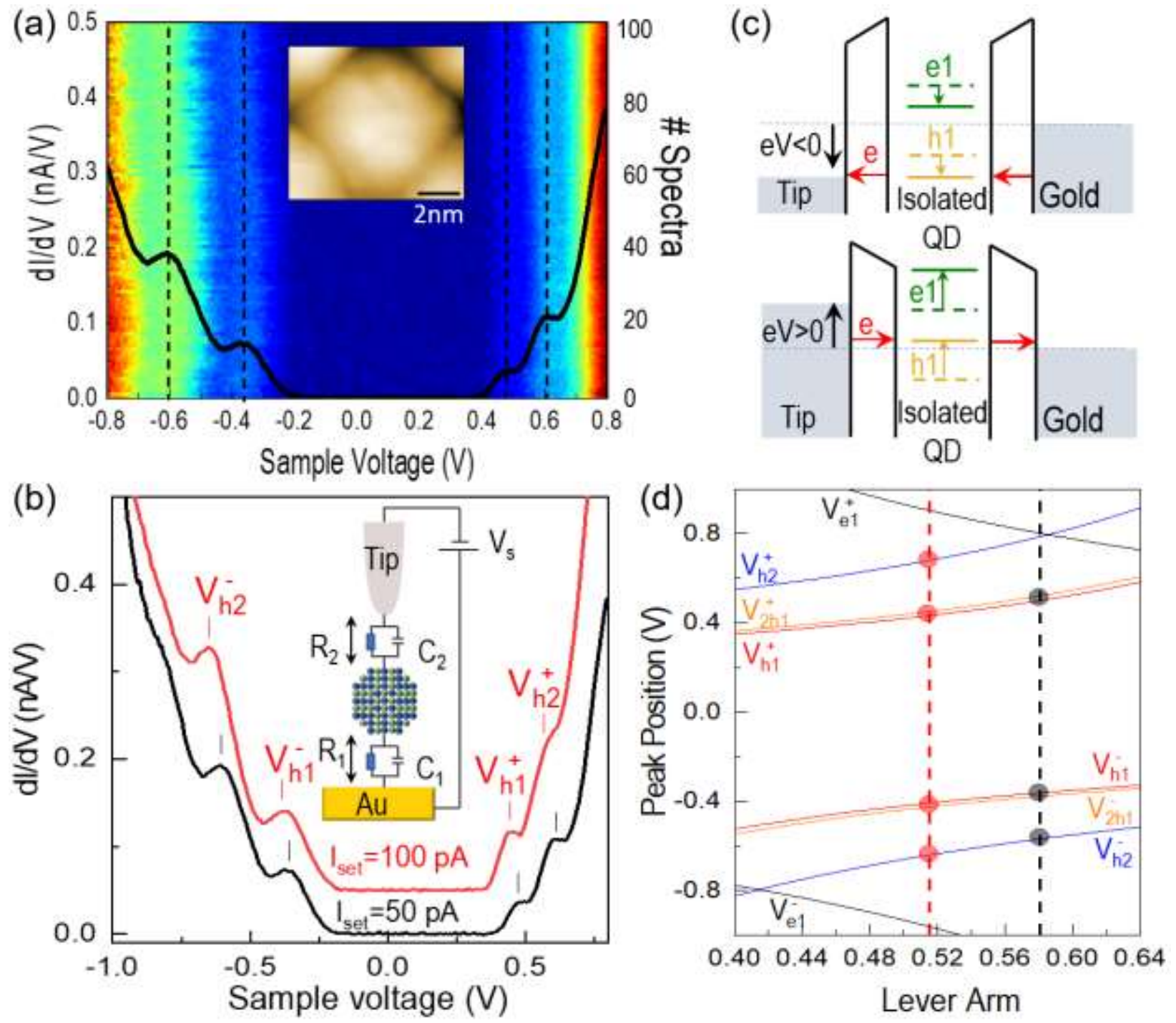

Figure 2. (a) Average dI/dV spectrum of an individual PbSe QD in an annealed superlattice on top of 100 consecutive $\mathrm{dI} / \mathrm{dV}$ curves concatenated into a color map $\left(\mathrm{V}_{\mathrm{S}}=+0.8 \mathrm{~V} ; \mathrm{I}_{\text {set }}=50 \mathrm{pA}\right)$. Inset: STM image of the QD. (b) dI/dV spectra measured at two different setpoint currents highlighting the shift of the peak position 
towards smaller bias as the current increases. The peaks labeled $\mathrm{V}^{-}{ }_{\mathrm{h} 1}, \mathrm{~V}^{-}{ }_{\mathrm{h} 2}, \mathrm{~V}^{+}{ }_{\mathrm{h} 1}, \mathrm{~V}^{+}{ }_{\mathrm{h} 2}$ correspond to the tunneling of holes through the ground and first excited states at negative and positive bias respectively. Inset: Schematic and equivalent circuit diagram of the double barrier tunnel junction for an isolated QD. The capacitance and the resistance of both barriers are labeled $\mathrm{C}_{1}, \mathrm{C}_{2}, \mathrm{R}_{1}, \mathrm{R}_{2}$. (c) Energy band diagrams of the system for a hole transport at negative (upper diagram) and positive (lower diagram) bias respectively. The Fermi level of the gold sample (dotted line) is used as a reference. Its proximity with the hole ground state $\left(h_{1}\right)$ at zero bias ensures the contribution of this state at both negative and positive biases, instead of the electron ground state $\left(e_{1}\right)$. (d) Variation of the peak position as a function of the lever arm $\eta$ in the limited range encompassing the experimental values of the level arms (vertical dotted line) deduced from (b). $\mathrm{V}^{-}{ }_{\mathrm{e} 1}, \mathrm{~V}^{-}{ }_{\mathrm{h} 2}, \mathrm{~V}^{-}{ }_{2 \mathrm{~h} 1}, \mathrm{~V}^{+}{ }_{\mathrm{el}}, \mathrm{V}^{+}{ }_{\mathrm{h} 2}, \mathrm{~V}^{+}{ }_{2 \mathrm{~h} 1}$ correspond to the variation of the expected peak for a tunneling involving the electron ground state, the hole first excited state and the hole ground state with a hole already in the QD at negative and positive bias respectively.

The acquisition of hundreds of successive curves on a single QD reveals a good reproducibility in the $\mathrm{dI} / \mathrm{dV}$ characteristics, demonstrating the positive impact of annealing on the stability of the tunneling conditions. Resonances are seen at positive and negative bias (Figure 2a). Assigning the origin of the resonances of colloidal QDs probed in STS is, however, not straightforward as they do not necessarily involve the tunneling of opposite charge carriers at positive and negative bias respectively. ${ }^{27,30}$ To resolve this issue, it is convenient to analyze the set-point current dependence of the $\mathrm{dI} / \mathrm{dV}$ curves and observe the direction of the peak shift in the tunneling spectra. Typically, shifts towards lower (higher) voltage values upon increasing set-point currents are characteristic for hole (electron) levels. Focusing on the QD seen in the inset of Figure $2 \mathrm{a}$, the comparison of the peak position in two spectra acquired at $50 \mathrm{pA}$ and $100 \mathrm{pA}$, respectively shows a clear shift of all resonances towards lower values as the set-point current increases. QDs exhibiting a ZCR unambiguously show this behavior, indicating that hole states are probed on both 
sides of the ZCR. It is caused by a Fermi level positioned below midgap in the superlattice, pointing to a $p$-type doping, in agreement with the transport measurements performed on similar superlattices. $^{24}$

Although a unipolar transport of holes cannot provide the band gap of individual QDs, because the same energy level is measured on both sides of the ZCR, it gives direct access to the distribution of the potential in the Double Tunneling Barrier Junction (DTBJ) junction, with important implications for the examination of the linewidth of the resonances. The DTBJ can be modelled with two capacitors, $\mathrm{C}_{1}$ and $\mathrm{C}_{2}$, connected in series, as illustrated in the inset of Figure 2b. The applied voltage to the gold substrate, Vs, is then distributed across the junctions. The ratio between the potential drop across the QD-tip junction and Vs is defined as the lever arm $\eta=$ $\mathrm{V}_{\mathrm{QD}} / \mathrm{Vs}=\mathrm{C}_{1} /\left(\mathrm{C}_{1}+\mathrm{C}_{2}\right)$, where $\mathrm{V}_{\mathrm{QD}}$ is the potential of the QD. For a unipolar transport of holes, the lever arm is directly obtained from the knowledge of the negative and positive voltages, $\mathrm{V}_{\mathrm{h} 1}{ }^{-}$and $\mathrm{V}_{\mathrm{h} 1}{ }^{+}$, at which the hole resonances occur: $\eta=\mathrm{V}_{\mathrm{h} 1}{ }^{+} /\left(\mathrm{V}_{\mathrm{h} 1}{ }^{+}-\mathrm{V}_{\mathrm{h} 1}{ }^{-}\right) .{ }^{27,30}$ For the QD shown in Figure 2, we find $\eta=0.58$ and 0.51 for set-point currents of 50 and $100 \mathrm{pA}$, respectively.

Writing the energy of the hole level relative to the Fermi level $h_{1}$, we can deduce the variation of the bias $\mathrm{Vh}_{\mathrm{hl}}{ }^{-}$and $\mathrm{Vh}_{\mathrm{hl}}{ }^{+}$as a function of the lever arm based on the following equations:

$$
\begin{aligned}
& V_{h_{1}}^{-}=\frac{h_{1}-\Sigma_{h}}{e \eta} \\
& V_{h_{1}}^{+}=-\frac{h_{1}-\Sigma_{h}}{e(1-\eta)}
\end{aligned}
$$

where $e$ is the elementary charge and $\Sigma_{\mathrm{h}}$ is the polarization energy of the hole that explicitly depends on the external dielectric constant and the size of the QD. ${ }^{30}$ By considering a perfectly cubic QD having in-plane epitaxial connection with its 4 neighbours, the rest of its surface being 
surrounded by vacuum, one estimates $\varepsilon_{\text {out }}$ to be $4 / 6$ of the PbSe dielectric constant, namely 151 . However, one has to take into account the truncated shape of the PbSe QDs that induces a necking and reduces the connection area between the QDs. A necking width of half the QD edge length is deduced from the STEM images, in agreement with previous reports, ${ }^{24,31}$ yielding $\varepsilon_{\text {out }} \sim 75$. Due to the high value of the external dielectric constant, the polarization energy is on the order of a few $\mathrm{meV}$ at most and therefore negligible compared to $h_{1}$. When the lever arm is one, $\mathrm{V}_{\mathrm{h} 1}{ }^{-}$corresponds to $h_{1}$. When the lever arm decreases, $\mathrm{V}_{\mathrm{h} 1}{ }^{-}$and $\mathrm{V}_{\mathrm{h} 1}{ }^{+}$are inversely proportional to $\eta$ and $1-\eta$, as shown in Figure 2d.

If we assume that the electronic levels of the QD are weakly coupled with the states of the QD neighbor, then the electronic structure of individual QDs in the superlattice can be considered close to the one of isolated QDs. Knowing the diameter of the QD from the topographic STM image, tight binding calculations provide a good description of their band structure. ${ }^{32}$ For instance, the theoretical band gap of a QD with a diameter of $5.6 \mathrm{~nm}$ is $0.67 \mathrm{eV},{ }^{33}$ implying the electron level $e_{1}$ to be positioned $0.47 \mathrm{eV}$ above the Fermi level. It also yields a separation of $0.12 \mathrm{eV}$ between the hole ground state $h_{1}$ and first excited state $h_{2}$. We note that the separation between the hole ground state $h_{1}$ and the same level with a hole already in the QD, $2 h_{1}$, is smaller than the instrumental resolution $(\sim 10 \mathrm{meV})$ due to the high dielectric constant in the superlattice. The corresponding resonances $\mathrm{V}_{\mathrm{el}}{ }^{+}, \mathrm{V}_{\mathrm{e} 1}{ }^{-}, \mathrm{V}_{\mathrm{h} 2}{ }^{-}, \mathrm{V}_{\mathrm{h} 2}{ }^{+}, \mathrm{V}_{2 \mathrm{~h} 1}{ }^{-}$and $\mathrm{V}_{2 \mathrm{~h} 1}{ }^{+}$can then be plotted as a function of the lever arm, as shown in Figure 2d. In the range of the lever arms deduced from the spectroscopic measurements, $\mathrm{V}_{\mathrm{el}}{ }^{+}, \mathrm{V}_{\mathrm{el}}{ }^{-}$are clearly higher and lower than $\mathrm{V}_{\mathrm{h} 1}{ }^{+}$and $\mathrm{V}_{\mathrm{h} 1}{ }^{-}$, consistent with the finding of a unipolar hole transport. Then, the second resonance observed at positive and negative bias complies well with the contribution of the first excited state of the QD valence band. We note that the assumption of weakly coupled QDs is valid for spectra with well-resolved peaks 
in the valence band. In case of stronger coupling, the density of states is modified. As discussed below, the QD band gap becomes smaller and cannot be correlated to the size of individual QDs. However, the observation of a clear shift of the band-edge onset at positive bias along with the appearance of a better resolved peak with increasing setpoint current allows to assign this peak to $h_{1}$ (Figure S6).

Having identified the resonances, we now turn to the analysis of their linewidth. Interestingly, we observe in Figure $2 \mathrm{~b}$, that the resonance measured at $\mathrm{V}_{\mathrm{h} 1}{ }^{+}$appears narrower than the resonance measured at $\mathrm{V}_{\mathrm{h} 1}{ }^{-}$despite a lever arm around 0.5 , which should result in similar linewidths as the resonance involves the same energy level. Figure 3a shows another example of three spectra acquired on the three neighboring QDs, which exhibit broad resonances at negative bias and almost similar narrow resonances at positive bias. The observation of step-like function instead of well-resolved peaks at negative bias in the upper spectra recalls the broad resonances measured in arrays of electronically coupled QDs separated from each other with short ligands. ${ }^{14,15}$ However, in such arrays, the resonances were usually broader at positive bias, implying a stronger electronic coupling of the conduction band states. In order to understand the origin of the asymmetry measured in the linewidth between negative and positive bias in the epitaxially connected QD superlattice, the resonances of the spectra were fitted with Gaussian functions, as illustrated for the spectrum of Figure 3b, which was acquired on another QD. The linewidth of the resonance expressed in $\mathrm{meV}$ was then obtained from the normalization of the measured full width at half maximum (FWHM) by the lever arm. The inset of Figure 3b reports the FWHM of the hole ground state at positive and negative voltages for QDs where the resonance labelled $\mathrm{Vh}_{\mathrm{h}}{ }^{-}$could unambiguously be fitted with a Gaussian function. Interestingly, the linewidths are strikingly different when the first hole state is probed with a negative or a positive voltage. For comparison, 
we also reported the calculated FWHM for a $5.8 \mathrm{~nm}$ wide isolated PbSe QD surrounded by ligands, which takes into account the electron-phonon coupling, the intervalley coupling and the influence of the surface ligands. ${ }^{33}$ The linewidths at positive voltage, which are rather steady around $55 \mathrm{meV}$, correspond to the ones of isolated QDs. Conversely, the linewidths at negative voltage are more scattered (between 60 and $100 \mathrm{meV}$ ) and are systematically broader than the linewidth of isolated $\mathrm{PbSe}$ QDs. As the stability of the dI/dV spectra shown in Figure 2a rules out any broadening induced by spectral diffusion, we attribute the larger linewidth measured at negative bias to the electronic coupling of the hole ground states between the QDs.
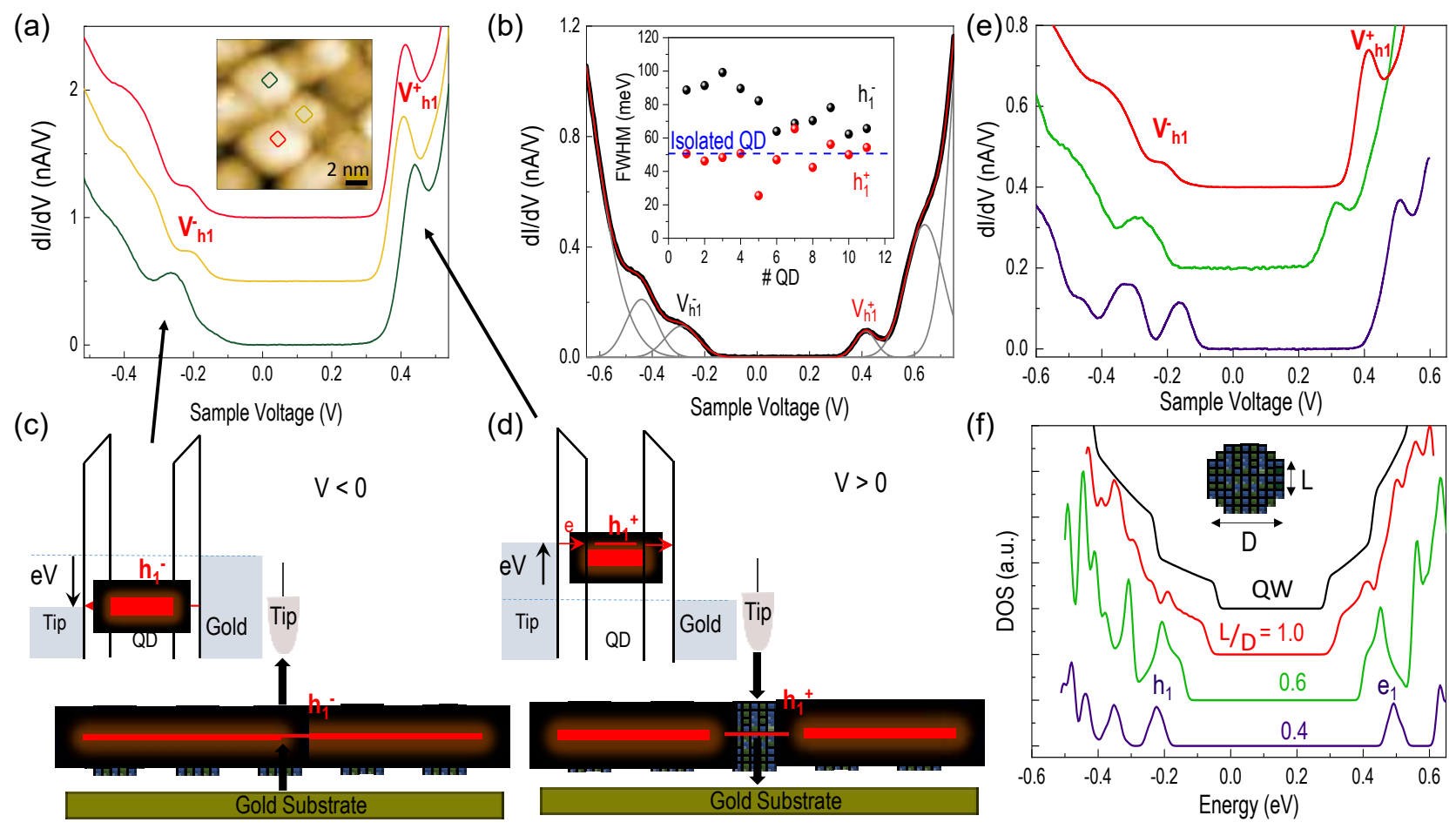

Figure 3. (a) $d I / d V$ spectra measured on three neighbor QDs shown in the inset $\left(V=+0.6 \mathrm{~V}\right.$; $\left.I_{\text {set }}=100 \mathrm{pA}\right)$. (b) A dI/dV spectrum fitted with Gaussian functions. The highest and lowest resonances measured at negative and positive bias are denoted $\mathrm{V}_{\mathrm{h} 1}{ }^{-}$and $\mathrm{V}_{\mathrm{h} 1}{ }^{+}$respectively. Inset: Normalized linewidth of the hole ground state $h_{1}$ measured at negative and positive bias for eleven QDs. The horizontal dash line shows the mean linewidth measured for isolated PbSe QDs surrounded by oleic acid ligands. (c) and (d) Energy band diagrams of the 
system for a hole transport at negative and positive bias respectively. The Fermi level of the gold sample (dotted line) is used as a reference. The hole ground state $\left(h_{1}\right)$ of the central QD is electronically coupled to the occupied hole states of the neighbor QDs at negative bias, but becomes decoupled from these states by the electrical field induced by the STM tip at positive bias. The black arrows highlight the successive transfer of the tunneling electron. (e) Example of dI/dV spectra measured on three different QDs, showing an increase of the resonance linewidth with stronger electronic coupling at negative bias and a barely modified linewidth of the resonance at positive bias. (f) Theoretical density of states of three QDs in a superlattice. The QDs have the same diameter D of $5.8 \mathrm{~nm}$ but different neck widths L. For comparison, the DOS of a $5.8 \mathrm{~nm}$-thick quantum well (QW) is also plotted.

We surmise that the asymmetry in the linewidth is induced by the proximity of the STM tip. Indeed, it has been well recognized that the applied potential between a STM tip and a semiconductor sample dropped in the semiconductor itself. Such an effect, known as a tip induced band bending, causes a rigid shift of the energy levels and is rather limited to the area below the apex. ${ }^{34,35}$ At negative voltage, the hole states are occupied. Due to the efficient screening of the PbSe QDs, the tip induced band bending is negligible and the measured linewidth reflects an electronic coupling of the QD with its next neighbors (Figure 3c). Conversely, at positive bias, the hole states of the QDs located below the STM are empty. The electrostatic screening is now poor and the tip induced band bending decouples the QD states from its next neighbors (Figure 3d). As a result, the STM tip measured the typical linewidth of isolated QDs. Sporadic charging in the $\mathrm{dI} / \mathrm{dV}$ spectra of a few individual QDs further supports this hypothesis. Figure 4a shows 60 consecutive spectra acquired on a QD where the reproducible $\mathrm{dI} / \mathrm{dV}$ curve (blue area) suddenly changes (red area) before returning to its initial characteristic (blue area). The reversibility and the transient character of the red area clearly indicate a charging event. Importantly, the charging effect 
causes a splitting of the resonance at positive bias only, when the hole state becomes decoupled. For this QD, a splitting $\Delta \mathrm{V}$ of $\sim 50 \mathrm{mV}$ implies $\varepsilon_{\text {out }}$ of about 35 , which is two times smaller than for the majority of the QDs in the superlattice. It suggests that this peculiar QD has a reduced number of epitaxial connections with its neighbors, so the extra carrier injected in the QD cannot be drained as efficiently than in the other case, thereby causing charging effects.

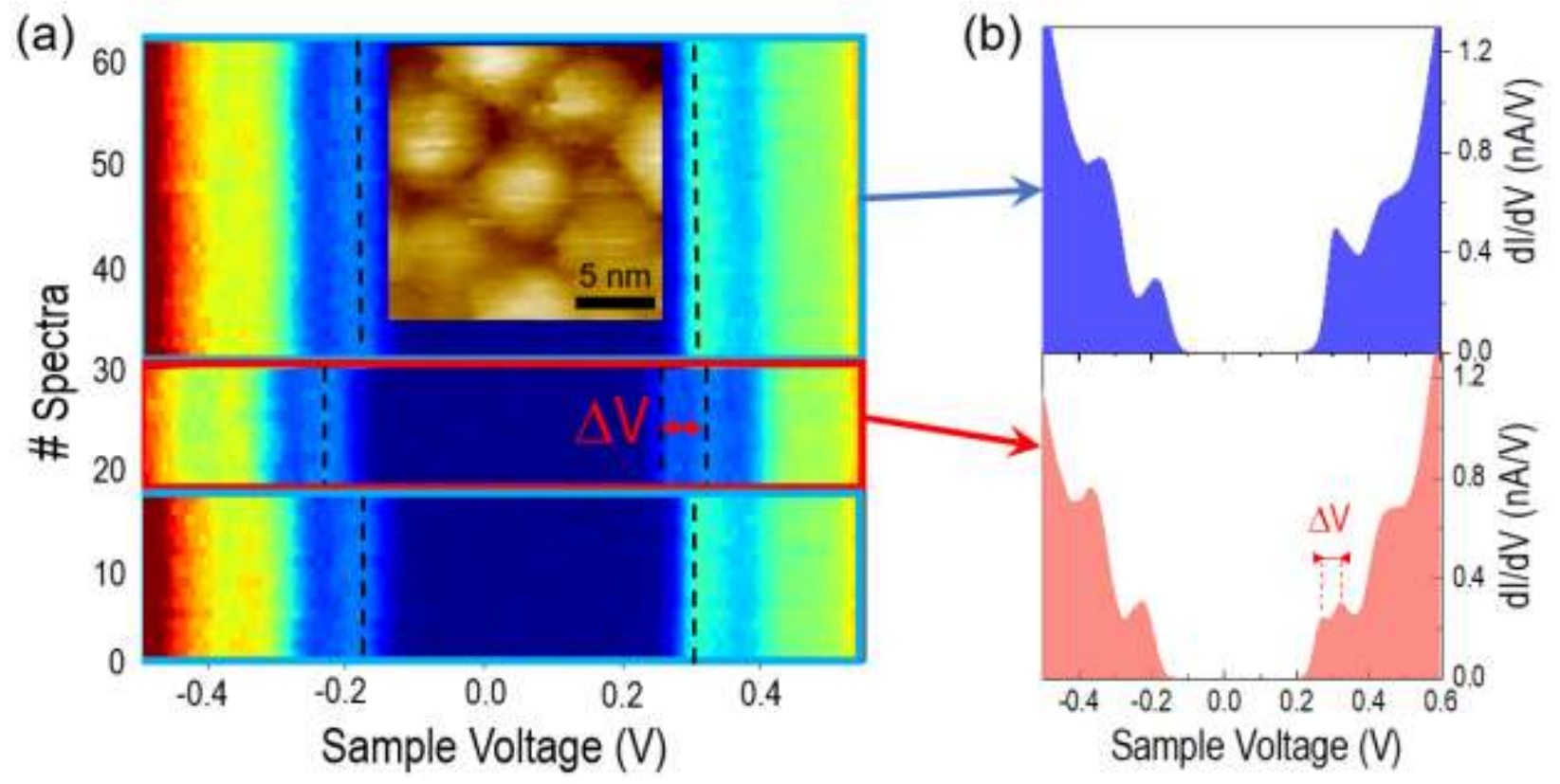

Figure 4. (a) Hundred consecutive dI/dV curves concatenated into a color map acquired on the central QD shown in the STM image of the inset. The highest and lowest resonances measured at negative and positive bias respectively are highlighted with vertical dashed segments. $\Delta \mathrm{V}$ indicates the bias separation in the range of spectra where the lowest resonance splits. (b) dI/dV spectra averaged in the blue (upper part) and red (lower part) domains, the latter one corresponding to a charging state.

Assuming that the majority of the QDs are epitaxially attached to their four neighbors, the density of states (DOS) of this system was theoretically modeled with tight binding calculations 
also. The QDs have a diameter $D=5.8 \mathrm{~nm}$. The neck between each pair of neighbor QDs is described by a cylinder of diameter $L$. Figure $3 \mathrm{f}$ shows the DOS for three different ratios between $L$ and $D$. For a ratio of 0.4 , the hole ground state appears as a peak, indicating a weak electronic coupling with the QD neighbors. Increasing the ratio leads to a broader resonance, with an onset that resembles the step like-function of a 2D electron gas. However, the formation of a genuine 2D density of states at a ratio of one is precluded because of the existence of periodic voids. These voids at the top and bottom of the compact grid are caused by the interconnected horizontal cylinders. Despite a temperature of $77 \mathrm{~K}$, which is not low enough to resolve the small valence peaks lying deeper in energy, the shape of distinct experimental spectra obtained at negative bias (Figure 3e) compares well with the theoretical DOS calculated for the valence band (Figure 3f). The DOS amplitude grows with increasing coupling and the background, which is better observed for the quantum well as the steps are not flat, is caused by the linear dispersion encountered in $\mathrm{PbSe}^{32,36}$ Therefore, the valence electron distribution is distorted in comparison with the one of isolated QDs. For most of the QDs, we estimated the necking size being 0.5 time the size of the QDs based on the STEM images. Such a ratio leads to a substantial coupling of the valence-band state, ensuring a hole localization length much larger than the QD diameter.

We now focus on the second set of spectra. Those spectra exhibit a resonance in the ZCR at positive bias. Based on previous STS studies of PbS QDs,${ }^{37}$ which showed a similar peak above the valence band, we attribute this resonance to the existence of in-gap states. While the QDs showing such in-gap resonance are sparsely distributed in the superlattice, the acquisition of hundreds of successive spectra on a QD with such a resonance (Figure 5a) reveals a good reproducibility in the $\mathrm{dI} / \mathrm{dV}$ characteristic (Figure $5 \mathrm{~b}$ ). This stability differs from the dynamical and random shift in the peak position of the tunneling spectra usually encountered on colloidal 
QDs with trap states, ${ }^{38,39}$ suggesting a significant reduction of the retention of spurious charges when the QDs are epitaxially connected. Based on previous structural and chemical analysis of the superlattice ${ }^{24}$ we suspect the trap states to be caused by interfacial defects or unpassivated atomic sites on the unbound facets. Indeed, the interfaces between the QDs are known to contain structural defects, vacancies and dislocations, despite the annealing of the superlattice. Furthermore, we cannot rule out the inadvertent desorption of ligands from the $\{111\}$ facets upon annealing. In order to discriminate between the different possible origins of the in-gap states, the remaining $\mathrm{Pb}$ oleate ligands were stripped off by exposing the sample to butylamine and then rinsing it with methanol. Such a treatment has a significant impact on the presence of the in-gap resonance: it appears on the majority of the QDs in the superlattice. For example, all six adjacent QDs visible in Figure $5 \mathrm{~b}$ show the in gap-resonance in the spectra of Figure $5 \mathrm{~d}$, after the removal of the ligands from the unbound facets. Therefore, we assign the origin of the in-gap states to the surface chemistry of the QDs and not to interfacial defects in the neck.

(a)

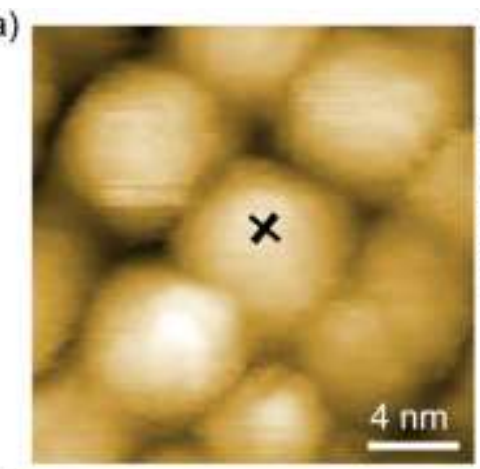

(b)

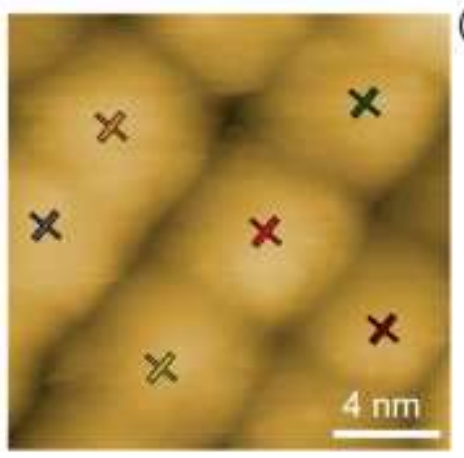

(c)

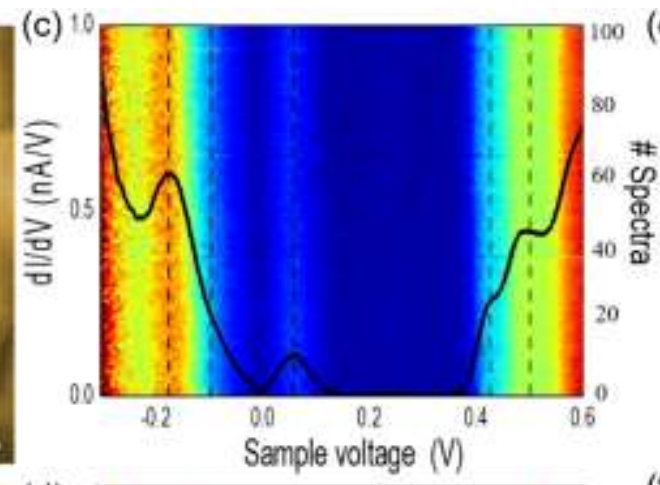

(d)

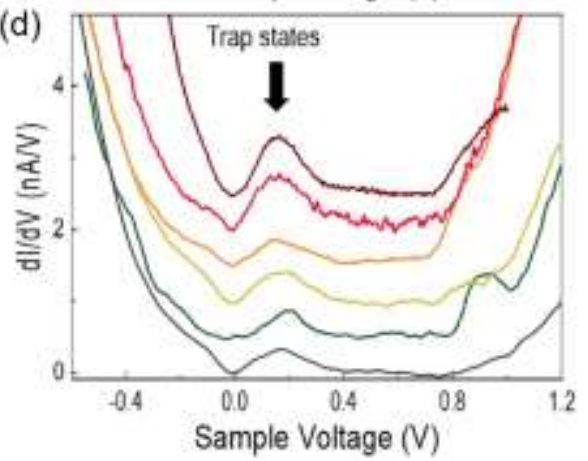

(e)

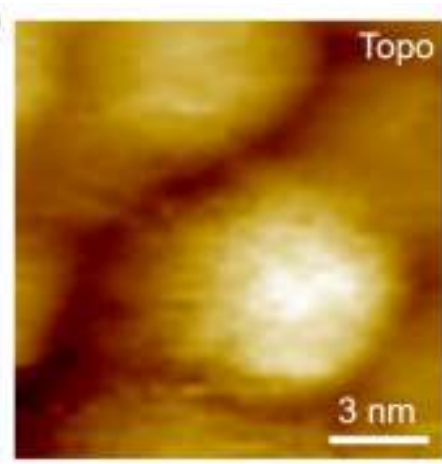

(f)

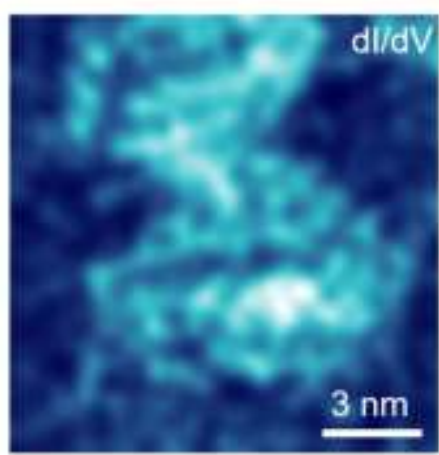


Figure 5. (a) STM image of the superlattice where the central QD exhibits in-gap states in the tunneling spectrum shown in (c). This spectrum is superimposed to 100 consecutive $\mathrm{dI} / \mathrm{dV}$ curves concatenated into a color map $\left(\mathrm{V}_{\mathrm{S}}\right.$ $=+0.7 \mathrm{~V}$; $\mathrm{I}_{\mathrm{set}}=200 \mathrm{pA}$ ). (b) STM image of a superlattice deposited on a $p$-type degenerate Si substrate where the $\mathrm{Pb}$-oleate ligands were stripped off prior to the experiment. The majority of the QDs in the superlattice exhibits in-gap states in tunneling spectroscopy, as shown in (d) $\left(V_{S}=+1.4 V\right.$; $\left.I_{\text {set }}=100 \mathrm{pA}\right)$. (e) STM image of two adjacent QDs with in-gap states and (f) related differential conductance map measured at the peak energy of the trap states.

Due to the depth of the in-gap states in the band gap of the defective QDs, the transfer rate of charge carriers injected into or escaping from these states is usually asymmetric. Indeed, the small localization of the wave function for a deep level in the band gap of PbSe prevents its strong coupling with either the tip states or the substrate states depending on its vertical position in the DTBJ. ${ }^{37}$ Moreover, as the preservation of ligands on the $\{111\}$ facets limits the number of QDs with in-gap states in the superlattice, electron hopping between adjacent QDs is not efficient. Both should lead to charging effects with clear shifts of the resonance with the bias. ${ }^{38,39}$ However, as already mentioned, the acquisition of hundreds of consecutive spectra reveals a good reproducibility in the $\mathrm{dI} / \mathrm{dV}$ characteristics (Figure 5c). An explanation for this inconsistency can be obtained from the examination of the electron distribution mapped with spatially resolved tunneling spectroscopy. At a bias corresponding to the energy of the in-gap states, the differential conductance shows a bright contrast which extends over the whole QD (Figure 5f). This is in clear contrast with the localization expected for a deep level at the surface of semiconductor materials. ${ }^{40,41}$ Our result suggests that the homogeneous contrast in the differential conductance map of Figure $5 \mathrm{f}$ reflects the presence of several defects on the unbound facets of a QD, each generating in-gap states. All together, they ensure an efficient transfer of the tunneling electron to 
the gold substrate via electron hopping at the surface of the defective QD, accounting for the absence of any charging effect.
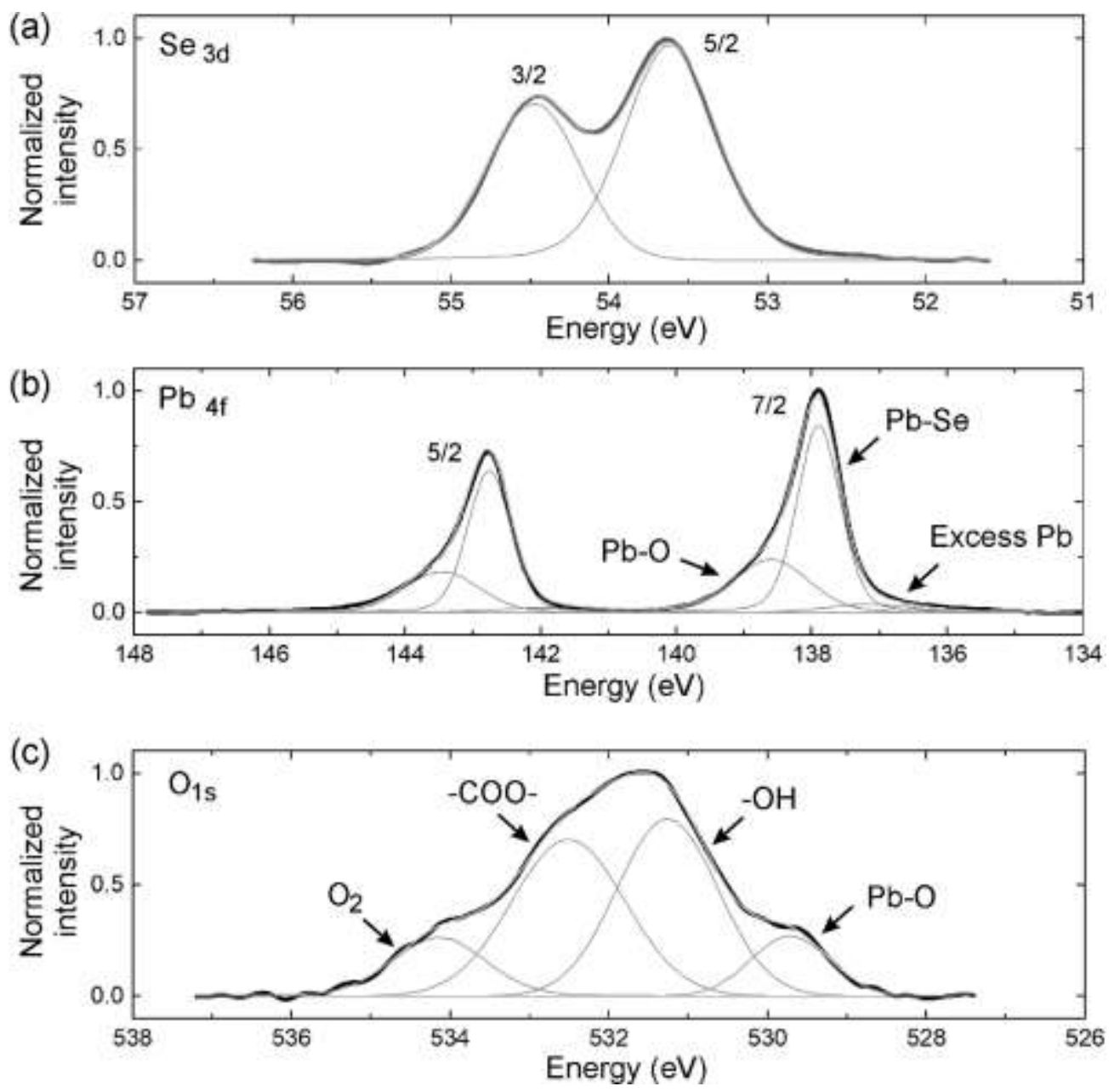

Figure 6. Photoelectron spectra of (a) $\mathrm{Se} 3 d$, (b) $\mathrm{Pb} 4 f$ and (c) $\mathrm{O} 1 s$ core levels measured on the 2D epitaxially connected and annealed PbSe QD superlattice deposited on the gold surface. The thin lines correspond to fits obtained with the parameters given in Table S1, SI.

To study the nature of the defects which are located at the surface of the defective QDs and able to generate such multiple in-gap states, we carried out X-ray photoemission spectroscopic 
(XPS) measurements. While the examination of the Se $3 d$ core level for the superlattice yields a branching ratio consistent with the literature ${ }^{42}$ and does not show any sign of oxidation (Figure 6a), fitting the $\mathrm{Pb} 5 d$ core level requires three doublets (Figure 6b). Based on previous XPS analyses of PbSe QDs, ${ }^{42}$ we identify the three components as follows: the central peak corresponds to the bulk component of $\mathrm{Pb}$ atoms bound to $\mathrm{Se}$; the small shoulder visible at low binding energy is caused by excess of unbound surface $\mathrm{Pb}$ atoms; finally, the broad shoulder at high binding energy arises from $\mathrm{PbO}$ species. These species also appear in the XPS spectrum of the $\mathrm{O} 1 \mathrm{~s}$ core level in three different forms (Figure 6c). The two major contributions arise from the $-\mathrm{COO}-$ groups of the oleate ligands $(532.5 \mathrm{eV})$ and from the $-\mathrm{OH}$ groups $(531.3 \mathrm{eV})$, consistent with the hydroxylation of the $\{111\}$ facets of PbS QDs passivated with oleic acid. ${ }^{43}$ The third one, at the lowest binding energy of $529.7 \mathrm{eV}$, is attributed to unpassivated $\mathrm{Pb}$ atoms. These atoms react with oxygen leading to $\mathrm{PbO}$ and $\mathrm{PbSeO}_{3}$ species. ${ }^{44}$ Interestingly, a fourth component is measured at a much higher binding energy (534.1 eV), similar to the one obtained on PbS QDs capped with 1,2ethanedithiol or treated with hydrazine and exposed to molecular oxygen.$^{45}$ Thus, the XPS results indicate that molecular oxygen is present at the surface of the superlattice, which could explain the formation of in-gap states. When additional $\mathrm{O}_{2}$ molecules, up to 10 molecules or so, adsorb, it has been shown that the energy levels of the in-gap states are almost unchanged as these states barely couple due to their localized character. ${ }^{45}$ The existence of multiple in-gap states, spread over the whole surface of a QD, is consistent with an efficient transfer of tunneling electrons to the gold substrate via electron hopping at the surface of the QD, accounting for the absence of any charging effect in $\mathrm{O}_{2}$-contaminated QDs. More importantly, acting collectively, the $\mathrm{O}_{2}$ molecules give rise to a state which is distributed over a large fraction of the QD surface and has a finite density in the center of the QD due to the small overlap of the individual states. Hence, the presence of multiple 
$\mathrm{O}_{2}$ molecules, that adsorb on unpassivated atomic sites of the $\mathrm{PbSe} \mathrm{QD}$ facets during the brief airtransfer of the superlattices, explains the homogeneous contrast in the differential conductance map of Figure $5 \mathrm{f}$. We point out that the physisorbed state of the $\mathrm{O}_{2}$ molecules at the surface of QDs is surprisingly robust as the superlattices were studied in UHV and were further annealed at $120^{\circ} \mathrm{C}$. An alternative to $\mathrm{O}_{2}$ molecules exists, namely neutral selenium adatoms ${ }^{46,47}$ But our XPS measurements do not give a hint to a high concentration of selenium adatoms when all the ligands have been removed. Also, we cannot rule out the formation of trap states resulting from the connectivity between QDs. When QDs are connected with a simple bridge instead of a neck, or when the necking involves $\mathrm{Pb}$ rich and Se rich facets, an asymmetric chemical environment and the formation of dipole appear. ${ }^{48}$ However, in this case, we would not expect a significant increase of the number of defective QDs with the removal of the ligands on the $\{111\}$ facets.

Finally, we emphasize the importance of generating extended conduction or valence states for raising the doping efficiency in the superlattice. Indeed, the defective QDs can trap electrons from the superlattice, leaving behind free holes in the extended valence states of the epitaxially connected PbSe QDs. Such an effect is captured by the spectroscopic measurements of Figure 1, where a reduction of the separation between the hole resonance $V_{h 1}{ }^{-}$and the Fermi level is visible for the defective QDs. Consistent with the literature, ${ }^{7,11}$ the valence and conduction states shift upward because of the negative charge carried by the QDs. We note that the calculation of the binding energy for a hydrogenic QD impurity in an epitaxially connected QD superlattice yields $10 \mathrm{meV}$ (Figure S7). This value is much smaller than the separation of $\sim 200 \mathrm{meV}$ measured between $E_{\mathrm{D}}$, the energy of the in-gap state, and $h_{1}$, pointing to a weak, but not negligible, doping efficiency. In order to estimate the efficiency of the trapping process, we have considered a twolevel model. Due to the small fluctuation of the energy levels in the superlattice and the inter-dot 
electronic coupling, both levels, $h_{1}$ and $E_{\mathrm{d}}$, were broadened with Gaussian functions. For $h_{1}$, including the spin and valley degeneracies, the density of states $\rho_{1}(\mathrm{E})$ is:

$$
\rho_{1}(E)=\frac{8}{\sigma_{1} \sqrt{2 \pi}} \exp \left(-\frac{\left(E-h_{1}\right)^{2}}{2 \sigma_{1}{ }^{2}}\right)
$$

with $\sigma_{1}$, the standard deviation, obtained from the experimental full width at half maximum divided by $2 \sqrt{ }(2 \ln 2)$. As to the density of states $\rho_{\mathrm{D}}(\mathrm{E})$ of the in-gap state, it writes:

$$
\rho_{D}(E)=\frac{n_{D}}{\sigma_{D} \sqrt{2 \pi}} \exp \left(-\frac{\left(E-E_{D}\right)^{2}}{2 \sigma_{D}{ }^{2}}\right)
$$

with $\sigma_{\mathrm{D}}$ the standard deviation and $n_{\mathrm{D}}$ the concentration of defective QDs in the superlattice. Using the Fermi-Dirac statistics, the Fermi energy is obtained by solving the charge neutrality condition considering that the total number of occupied states per QD is eight. At a temperature of $77 \mathrm{~K}$, for a separation of $200 \mathrm{meV}$ between both levels and a broadening of $70 \mathrm{meV}$, consistent with the experimental broadening, we find a Fermi level lying between $20 \mathrm{meV}$ and $40 \mathrm{meV}$ below $\mathrm{E}_{\mathrm{d}}$. This position, which does not vary significantly with the concentration of the defective QDs in the superlattice (Figure 7a), agrees well with the energy at which the resonances related to the in-gap states are measured in Figure 1f. It roughly yields a hole every thousand QDs (Figure 7b). Importantly, the doping efficiency strongly depends on the broadening. Assuming a concentration $n_{\mathrm{D}}$ of $0.6 \times 10^{12} \mathrm{~cm}^{-2}$, the number of free holes supplied to the superlattice decreases by three orders of magnitude when the broadening is reduced to $30 \mathrm{meV}$ (inset of Figure $7 \mathrm{~b}$ ). Therefore, albeit weak, the electronic coupling between the PbSe QDs is key in accounting for a measurable doping in the superlattice at room temperature, ${ }^{24}$ where a broadening of $70 \mathrm{meV}$ yields a doping level around $2.7 \times 10^{10}$ hole. $\mathrm{cm}^{-2}$. 

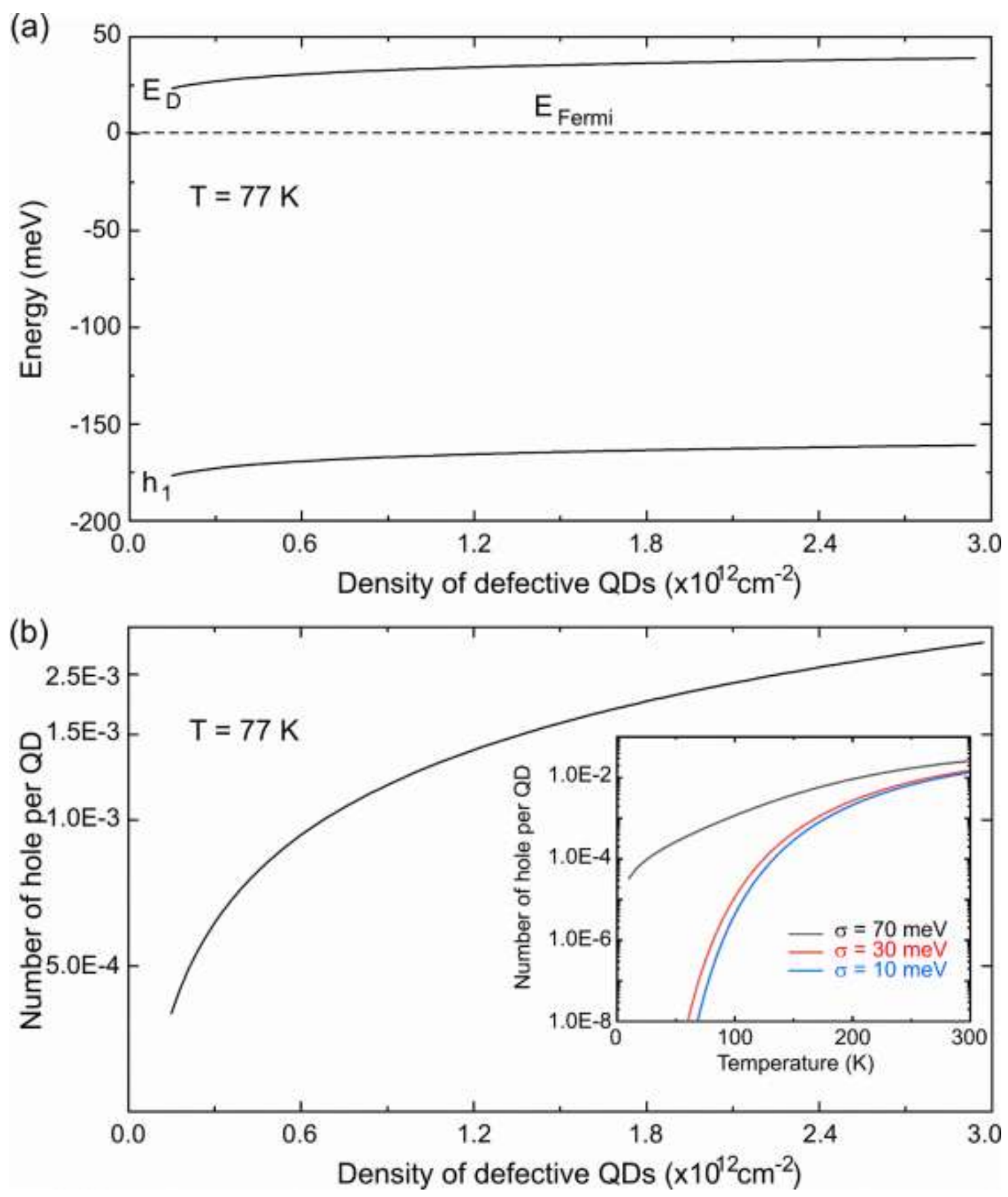

Figure 7. (a) Influence of the density of defective QDs in the superlattice, $n_{\mathrm{D}}$, on the position of the Fermi level between the hole ground state $h_{1}$ and the in-gap state $E_{\mathrm{D}}$. (b) Variation of the number of hole per QD as a function of the density of defective QDs. Inset: Variation of the number of hole per QD as a function of the temperature for a density of defective QDs of $0.6 \times 10^{12} \mathrm{~cm}^{-2}$. The calculations were performed for an energy separation between $h_{1}$ and $E_{\mathrm{D}}$ of $200 \mathrm{meV}$. The same standard deviation $\sigma$ was assumed for both levels. 


\section{CONCLUSION}

In summary, we have used low temperature STM to unveil the electronic properties of individual PbSe QDs that are epitaxially connected through their (100) facets and form a square superlattice. The QDs show a systematic p-doping with an electronic coupling of their valence states. This doping is caused by a minority of defective QDs acting as acceptor dopants in a medium with a dielectric constant nearly one order of magnitude higher than in standard selfassemblies, which considerably reduces the charging energy down to a few meV. The experiments have revealed the importance of the Pb-oleate ligand removal at the surface of the QDs to induce in-gap states at the origin of the doping. Although the formation of in-gap states is tentatively assigned to molecular oxygen, further research is needed to determine the physical origin of the trap and take control of the incorporation of acceptor QDs in the superlattice.

\section{METHODS}

Synthesis of Colloidal PbSe QDs. Monodisperse batches of PbSe QDs with a diameter of $5.8 \mathrm{~nm}$ were synthesized by reacting lead oleate and trioctylphosphine selenium following the approach described in Ref. 24.

Formation of Superlattices of Interconnected PbSe QDs. 2D superlattices of interconnected PbSe QDs were formed at a liquid-air interface by casting the amount of PbSe QDs needed to form a closepacked monolayer onto an ethylene glycol liquid substrate, as described in Ref. 24. The formation of a QD superlattice with square symmetry was triggered by the addition of aniline, a mild lead oleate stripping agent, to the subphase. After reacting for $30 \mathrm{~min}$, the floating QD 
superlattice was transferred via Langmuir-Schaeffer deposition to a gold substrate or a TEM grid. The reaction and the superlattice deposition were performed in a nitrogen-filled glove box and the samples were finally loaded into a sealed container to avoid air exposure during the transfer between Ghent and Lille.

Tight-Binding Calculations. The electronic structure of PbSe QDs with infinite lateral sizes was calculated using a sp ${ }^{3} \mathrm{~d}^{5} \mathrm{~s}^{*}$ tight-binding model as described in Ref. 24.

Scanning tunnelling microscopy. The samples were quickly taken from the container in air to be quickly loaded into an ultrahigh vacuum system with a base pressure of $1 \times 10^{-10}$ Torr, consisting of a preparation chamber and a low temperature scanning tunnelling microscope. After their annealing in the preparation chamber, they were examined with tungsten tips at a temperature of $77 \mathrm{~K}$. Tunneling spectroscopic measurements were performed at constant tip-sample separation. The dynamic conductance was measured with a lock-in amplifier using typically $7 \mathrm{mV}$ pk-pk modulation at $480 \mathrm{~Hz}$ on the bias voltage.

Scanning Transmission Electron Microscopy. A FEI Titan Themis 300 microscope equipped with a probe aberration corrector, operated at $200 \mathrm{kV}$, was used to acquire (HR)STEM images. The probe size was set to $0.1 \mathrm{~nm}$ with a convergence semiangle of $22.5 \mathrm{mrad}$. Collection angle of the HAADF detector was in the range $80-150$ mrad. Contrasts in an HAADF image is proportional to $Z \sim 1.7-2$, meaning that the bright contrast indicates relatively heavy atomic composition.

X-ray photoelectron spectroscopy. The XPS measurements were performed with a monochromatic $\mathrm{Al} \mathrm{K \alpha} \mathrm{X}$-ray source and an analyser pass energy of $12 \mathrm{eV}$. Under these conditions, the overall resolution as measured from the full width at half-maximum (fwhm) of the $\mathrm{Ag} 3 d 5 / 2$ line is $0.55 \mathrm{eV}$. The binding energy scale was calibrated using the $\mathrm{Au} 4 f 7 / 2$ line at $84.0 \mathrm{eV}$. The 
acceptance angle of the analyser was set to $14^{\circ}$, and the angle between the incident X-rays and the analyser was $90^{\circ}$. The detection angle of the photoelectrons was $45^{\circ}$, as referenced to the sample surface. The intensities of the various XPS core levels were measured as the peak area after standard background subtraction according to the Shirley procedure. For the core level decomposition, we used Voigt functions and a least squares minimization procedure. The different components were modelled with the same parameters; that is, the Gaussian and Lorentzian broadenings were kept fixed for each component of a given core level.

\section{ASSOCIATED CONTENT}

\section{Supporting information}

The Supporting Information is available free of charge at :

Additional details regarding structural analysis obtained from TEM experiments, STS of unannealed QDs, STM measurements of the buckling effects, two examples of tunneling spectra highlighting a hole transport, theoretical calculations of the binding energy of a hydrogenic QD impurity, fitting parameters of the core levels measured with XPS.

\section{AUTHOR INFORMATION}

\section{Corresponding Author}

*E-mail: louis.biadala@iemn.fr

*E-mail: bruno.grandidier@univ-lille.fr 


\section{Author Contributions}

The manuscript was written through contributions of all authors. All authors have given approval to the final version of the manuscript.

\section{ACKNOWLEDGMENT}

This study was financially supported by the European Community’s H2020 Program (Grant No. PITN-GA-2016-722176, “Indeed” Project), the EQUIPEX program Excelsior (Grant No. ANR11-EQPX-0015), IEMN PCMP-PCP platform of the RENATECH network, the Agence National de la Recherche (Grant No. ANR-19-CE09-0022, "TROPICAL” Project) and I-SITE ("PRIVET" project). Z.H. acknowledges funding from Ghent University (GOA 01G01019).

\section{REFERENCES}

(1) Ho, J. C.; Yerushalmi, R.; Jacobson, Z. A.; Fan, Z.; Alley, R. L.; Javey, A. Controlled Nanoscale Doping of Semiconductors via Molecular Monolayers. Nat. Mater. 2008, 7, 62-67.

(2) Perea, D. E.; Hemesath, E. R.; Schwalbach, E. J.; Lensch-Falk, J. L.; Voorhees, P. W.;

Lauhon, L. J. Direct Measurement of Dopant Distribution in an Individual Vapour-Liquid-Solid Nanowire. Nat. Nanotechnol. 2009, 4, 315-319.

(3) Zwanenburg, F. A.; Dzurak, A. S.; Morello, A.; Simmons, M. Y.; Hollenberg, L. C.; Klimeck, G.; Rogge, S.; Coppersmith, S. N.; Eriksson, M. A. Silicon Quantum Electronics. Rev. Mod. Phys. 2013, 85, 961.

(4) Stavrinadis, A.; Konstantatos, G. Strategies for the Controlled Electronic Doping of Colloidal Quantum Dot Solids. Chem. Phys. Chem. 2016, 17, 632-644. 
(5) Kagan, C. R.; Lifshitz, E.; Sargent, E. H.; Talapin, D. V. Building Devices from Colloidal Quantum Dots. Science 2016, 353, 885.

(6) Panfil, Y. E.; Oded, M.; Banin, U. Colloidal Quantum Nanostructures: Emerging Materials for Display Applications. Angew. Chem. Int. Ed. 2018, 57, 4274-4295.

(7) Mocatta, D.; Cohen, G.; Schattner, J.; Millo, O.; Rabani, E.; Banin, U. Heavily Doped Semiconductor Nanocrystal Quantum Dots. Science 2011, 332, 77-81.

(8) Sahu, A.; Kang, M. S.; Kompch, A.; Notthoff, C.; Wills, A. W.; Deng, D.; Winterer, M.; Frisbie, C. D.; Norris, D. J. Electronic Impurity Doping in CdSe Nanocrystals. Nano Lett. 2012, $12,2587-2594$.

(9) Yu, D. ; Wang, C. ; Guyot-Sionnest, P. n-Type Conducting CdSe Nanocrystal Solids. Science 2003, 300, 1277-1280.

(10) Talapin, D. V.; Murray, C. B. PbSe Nanocrystal Solids for n-and p-Channel Thin Film Field-Effect Transistors. Science 2005, 310, 86-89.

(11) Bekenstein, Y.; Vinokurov, K.; Keren-Zur, S.; Hadar, I.; Schilt, Y.; Raviv, U.; Millo, O.; Banin, U. Thermal Doping by Vacancy Formation in Copper Sulfide Nanocrystal Arrays. Nano Lett. 2014, 14, 1349-1353.

(12) Tang, J.; Brzozowski, L.; Barkhouse, D. A. R.; Wang, X.; Debnath, R.; Wolowiec, R.; Palmiano, E; Levina, L.; Pattantyus-Abraham, A. G.; Jamakosmanovic, D; Sargent, E. H. Quantum Dot Photovoltaics in the Extreme Quantum Confinement Regime: the Surface-Chemical Origins of Exceptional Air-and Light-Stability. ACS Nano 2010, 4, 869-878.

(13) Kagan, C. R.; Murray, C. B. Charge Transport in Strongly Coupled Quantum Dot Solids. Nat. Nanotechnology 2015, 10, 1013-1026. 
(14) Liljeroth, P.; Overgaag, K.; Urbieta, A.; Grandidier, B.; Hickey, S. G.; Vanmaekelbergh, D. Variable Orbital Coupling in a Two-Dimensional Quantum-Dot Solid Probed on a Local Scale. Phys. Rev. Lett. 2006, 97, 096803.

(15) Steiner, D.; Aharoni, A.; Banin, U.; Millo, O. LevelS of InAs Quantum Dots in TwoDimensional Assemblies. Nano Lett. 2006, 6, 2201-2205.

(16) Cargnello, M.; Johnston-Peck, A. C.; Diroll, B. T.; Wong, E.; Datta, B.; Damodhar, D.; Doan-Nguyen, V. V. T.; Herzing, A. A.; Kagan, C. R.; Murray, C. B. Substitutional Doping in Nanocrystal Superlattices. Nature 2015, 524, 450-453.

(17) Jishkariani, D.; Elbert, K. C.; Wu, Y., Lee; J. D.; Hermes, M.; Wang, D.; van Blaaderen, A.; Murray, C. B. Nanocrystal Core Size and Shape Substitutional Doping and Underlying Crystalline Order in Nanocrystal Superlattices. ACS Nano 2019, 13, 5712-5719.

(18) Evers, W. H.; Schins, J. M.; Aerts, M.; Kulkarni, A.; Capiod, P.; Berthe, M.; Grandidier, B.; Delerue, C.; van der Zant, H. S. J.; van Overbeek, C.; Peters J. L.; Vanmaekelbergh, D.; Siebbeles, L. D. High Charge Mobility in Two-Dimensional Percolative Networks of PbSe Quantum Dots Connected by Atomic Bonds. Nat. Commun. 2015, 6, 1-8.

(19) Whitham, K.; Yang, J.; Savitzky, B. H.; Kourkoutis, L. F.; Wise, F.; Hanrath, T. Charge Transport and Localization in Atomically Coherent Quantum Dot Solids. Nat. Mater. 2016, 15, $557-563$.

(20) Balazs, D. M.; Matysiak, B. M.; Momand, J.; Shulga, A. G.; Ibáñez, M.; Kovalenko, M. V.;

Kooi, B. J.; Loi, M. A. Electron Mobility of $24 \mathrm{~cm}^{2} \mathrm{~V}^{-1} \mathrm{~s}^{-1}$ in PbSe Colloidal-Quantum-Dot Superlattices. Adv. Mater. 2018, 30, 1802265. 
(21) Zhao, Q.; Gouget, G.; Guo, J.; Yang, S.; Zhao, T.; Straus, D. B.; Qian, C.; Oh, N.; Wang, H.; Murray, C. B.; Kagan, C. R. Enhanced Carrier Transport in Strongly Coupled, Epitaxially Fused CdSe Nanocrystal Solids. Nano Lett. 2021, 21, 3318-3324.

(22) Salzmann, B. B.; van der Sluijs, M. M.; Soligno, G.; Vanmaekelbergh, D. Oriented Attachment: From Natural Crystal Growth to a Materials Engineering Tool. Acc. Chem. Res. 2021, 54, 787-797.

(23) Sandeep, C. S.; Azpiroz, J. M.; Evers, W. H.; Boehme, S. C.; Moreels, I.; Kinge, S.; Siebbeles L. D. A.; Infante, I.; Houtepen, A. J. Epitaxially Connected PbSe Quantum-Dot Films: Controlled Neck Formation and Optoelectronic Properties. ACS Nano 2014, 8, 11499-11511.

(24) Walravens, W.; Solano, E.; Geenen, F.; Dendooven, J.; Gorobtsov, O.; Tadjine, A.; Mahmoud, N.; Ding, P. P.; Ruff, J. P. C.; Singer, A.; Roelkens, G.; Delerue, C.; Detavernier, C.; Hens, Z. Setting Carriers Free: Healing Faulty Interfaces Promotes Delocalization and Transport in Nanocrystal Solids. ACS Nano 2019, 13, 12774-12786.

(25) Capiod, P.; van der Sluijs, M.; de Boer, J.; Delerue, C.; Swart, I.; Vanmaekelbergh, D. Electronic Properties of Atomically Coherent Square PbSe Nanocrystal Superlattice Resolved by Scanning Tunneling Spectroscopy. Nanotechnology 2021, 32, 325706.

(26) Koenraad, P. M.; Flatté, M. E. Single Dopants in Semiconductors. Nat. Mater. 2011, 10, $91-100$.

(27) Nguyen, T. H.; Habinshuti, J.; Justo, Y.; Gomes, R.; Mahieu, G.; Godey, S.; Nys, J. P.; Carrillo, S.; Hens, Z.; Robbe, O.; Turrell, S.; Grandidier, B. Charge Carrier Identification in Tunneling Spectroscopy of Core-Shell Nanocrystals. Phys. Rev. B 2011, 84, 195133. 
(28) Liljeroth, P.; van Emmichoven, P.; Hickey, S.; Weller, H.; Grandidier, B.; Allan, G.; Vanmaekelbergh, D. Density of States Measured by Scanning-Tunneling Spectroscopy Sheds New Light on the Optical Transitions in PbSe Nanocrystals. Phys. Rev. Lett. 2005, 95, 086801.

(29) Banin, U.; Millo, O. Tunneling and Optical Spectroscopy of Semiconductor Nanocrystals. Аnпu. Rev. Phys. Chem. 2003, 54, 465-492.

(30) Niquet, Y. M.; Delerue, C.; Allan, G.; Lannoo, M. Interpretation and Theory of Tunneling Experiments on Single Nanostructures. Phys. Rev. B 2002, 65, 1-14.

(31) Geuchies, J. J.; van Overbeek, C.; Evers, W. H.; Goris, B.; de Backer, A.; Gantapara, A. P.; Rabouw, F. T.; Hilhorst, J.; Peters, J. L.; Konovalov, O.; et al. In Situ Study of the Formation Mechanism of Two-Dimensional Superlattices from PbSe Nanocrystals. Nat. Mater. 2016, 15, $1248-1254$.

(32) Allan, G.; Delerue, C. Confinement Effects in PbSe Quantum Wells and Nanocrystals. Phys. Rev. B 2004, 70, 245321.

(33) Overgaag, K.; Vanmaekelbergh, D.; Liljeroth, P.; Mahieu, G.; Grandidier, B.; Delerue, C.; Allan, G. Electron-Phonon Coupling and Intervalley Splitting Determine the Linewidth of SingleElectron Transport through PbSe Nanocrystals. J. Chem. Phys. 2009, 131, 224510.

(34) Dombrowski, R.; Steinebach, C.; Wittneven, C.; Morgenstern, M.; Wiesendanger, R. TipInduced Band Bending by Scanning Tunneling Spectroscopy of the States of the Tip-Induced Quantum Dot on InAs (110). Phys. Rev. B 1999, 59, 8043.

(35) Feenstra, R. M.; Dong, Y.; Semtsiv, M. P.; Masselink, W. T. Influence of Tip-Induced Band Bending on Tunnelling Spectra of semiconductor surfaces. Nanotechnology 2006, 18, 044015. 
(36) Lannoo, M.; Prins, P.T.; Hens, Z.; Vanmaekelbergh, D.; Delerue, C. Universality of optical absorptance quantization in two-dimensional group-IV, III-V, II-VI, and IV-VI semiconductors Phys. Rev. B. 2022, in press.

(37) Zhang, Y.; Zherebetskyy, D.; Bronstein, N. D.; Barja, S.; Lichtenstein, L.; Schuppisser, D.; Wang, L.-W.; Alivisatos, A. P.; Salmeron, M. Charge Percolation Pathways Guided by Defects in Quantum Dot Solids. Nano Lett. 2015, 15, 3249-3253.

(38) Hummon, M. R.; Stollenwerk, A. J.; Narayanamurti, V.; Anikeeva, P. O.; Panzer, M. J.; Wood, V.; Bulović, V. Measuring Charge Trap Occupation and Energy Level in CdSe/ZnS Quantum Dots using a Scanning Tunneling Microscope. Phys. Rev. B 2010, 81, 115439.

(39) Peric, N.; Lambert, Y.; Singh, S.; Khan, A. H.; Franchina Vergel, N. A.; Deresmes, D.; Berthe, M.; Hens, Z.; Moreels, I.; Delerue, C.; Grandidier, B.; Biadala, L. Van Hove Singularities and Trap States in Two-Dimensional CdSe Nanoplatelets. Nano Lett. 2021, 21, 1702-1708.

(40) Berthe, M.; Stiufiuc, R.; Grandidier, B.; Deresmes, D.; Delerue, C.; Stiévenard, D. Probing the Carrier Capture Rate of a Single Quantum Level. Science 2008, 319, 436-438.

(41) Kislitsyn, D. A.; Mills, J. M.; Kocevski, V.; Chiu, S. K.; DeBenedetti, W. J. I.; Gervasi, C. F.; Taber, B. N.; Rosenfield, A. E.; Eriksson, O.; Rusz, J.; Goforth, A. M.; Nazin, G. V. Visualization and Spectroscopy of Defects Induced by Dehydrogenation in Individual Silicon Nanocrystals. J. Chem. Phys. 2016, 144, 241102.

(42) Habinshuti, J.; Capiod, P.; Nguyen, T. H.; Justo, Y.; Avila, F. J.; Asensio, M. C.; Bournel, F.; Gallet, J.JJ.; Vobornik, I.; Fujii, J.; Addad, A.; Lambert, Y.; Hens, Z.; Osherov, A.; Golan, Y.; Robbe, O.; Turell, S.; Grandidier, B. Phase Transformation of PbSe/CdSe Nanocrystals from CoreShell to Janus Structure Studied by Photoemission Spectroscopy. Phys. Rev. B 2013, 87, 184102. 
(43) Zherebetskyy, D.; Scheele, M.; Zhang, Y.; Bronstein, N.; Thompson, C.; Britt, D.;

Salmeron, A.; Alivisatos, P.; Wang, L. W. Hydroxylation of the Surface of PbS Nanocrystals Passivated with Oleic Acid. Science 2014, 344, 1380-1384.

(44) Tan, L.; Li, P.; Sun, B.; Chaker, M.; Ma, D. Stabilities Related to Near-Infrared Quantum Dot-based Solar Cells: the Role of Surface Engineering. ACS Energy Lett. 2017, 2, 1573-1585.

(45) Zhang, Y.; Zherebetskyy, D.; Bronstein, N. D.; Barja, S.; Lichtenstein, L.; Alivisatos, A. P.; Wang, L.-W.; Salmeron, M. Molecular Oxygen Induced In-Gap States in PbS Quantum Dots. ACS Nano 2015, 9, 10445-10452.

(46) Oh, S. J.; Berry, N. E.; Choi, J. H.; Gaulding, E. A.; Paik, T.; Hong, S. H.; Murray, C. B.; Kagan, C. R. Stoichiometric Control of Lead Chalcogenide Nanocrystal Solids to Enhance their Electronic and Optoelectronic Device Performance. ACS Nano 2013, 7, 2413-2421.

(47) Zherebetskyy, D.; Zhang, Y.; Salmeron, M.; Wang, L. W. Tolerance of Intrinsic Defects in PbS Quantum Dots. J. Phys. Chem. Lett. 2015, 6, 4711-4716.

(48) Li, H.; Zhitomirsky, D.; Dave, S.; Grossman, J. C. Toward the Ultimate Limit of Connectivity in Quantum Dots with High Mobility and Clean Gaps. ACS Nano 2016, 10, 606-614. 


\section{Supporting Informations}

\section{Quantum Dot Acceptors in Two-Dimensional}

\section{Epitaxially Fused PbSe Quantum Dot Superlattices}

Vincent Notot ${ }^{\dagger}$, Willem Walravens ${ }^{\|}$, Maxime Berthe ${ }^{\dagger}$, Nemanja Peric ${ }^{\dagger}$, Ahmed Addad ${ }^{\S}$, Xavier Wallart $^{\dagger}$, Christophe Delerue ${ }^{\dagger}$, Zeger Hens", Bruno Grandidier ${ }^{\dagger, *}$ and Louis Biadala, ${ }^{\dagger, *}$

${ }^{\dagger}$ Univ. Lille, CNRS, Centrale Lille, Univ. Polytechnique Hauts-de-France, JUNIA-ISEN, UMR 8520 - IEMN, F-59000 Lille, France.

${ }^{\S}$ Univ. Lille, CNRS, INRAE, Centrale Lille, UMR 8207 - UMET - Unité Matériaux et Transformations, F-59000, Lille, France

"Physics and Chemistry of Nanostructures, Ghent University, 9000 Ghent, Belgium 


\section{Defects in the as-grown in superlattice of PbSe QDs}

In Figure S1, a number of recurrent defects are shown, such as the missing connection between the dots (red arrow in Figure S1A). In addition, as highlighted in Figure S1B, the poor alignment of the atomic lattice of the QD during the formation of the superlattice can lead to the formation of dislocations. The atomic lattice orientation of the QDs on the right does not align with the atomic lattice of their left neighbors, leading to a dislocation and inducing some strain at the grain boundary. Moreover, the size dispersion can also be a factor of disorder as it may lead to void in the arrays and create missing connections between QDs (Figure S1C)

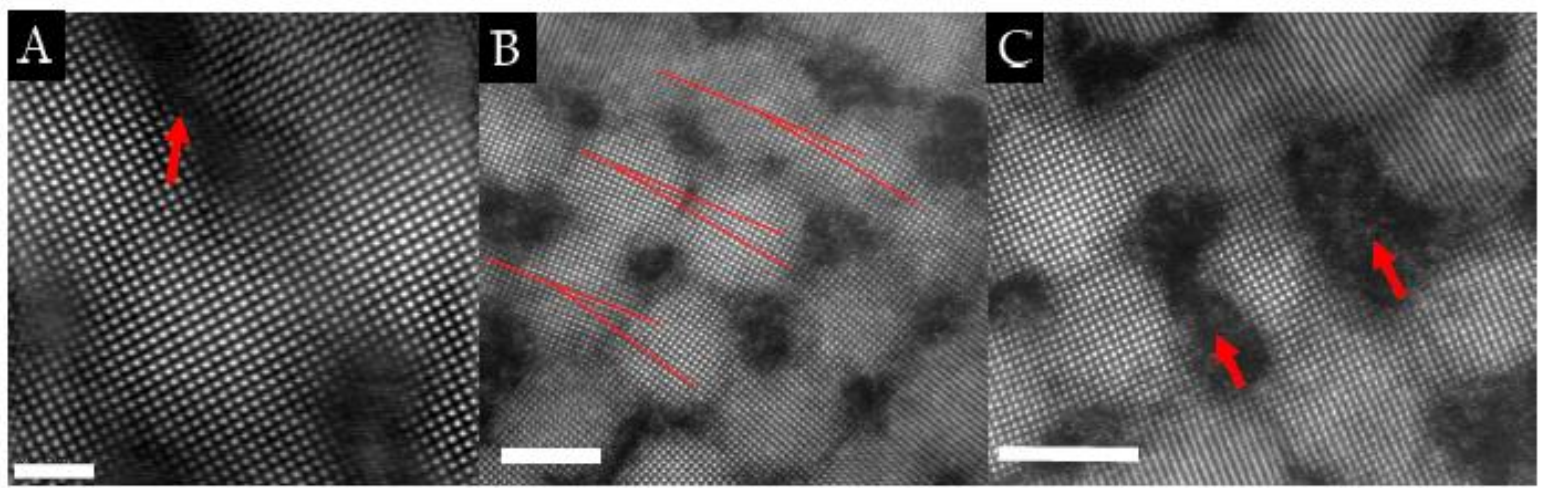

Figure S1: STEM High-angle annular dark-field imaging (HAADF) images of $5.8 \mathrm{~nm}$ PbSe QDs after aniline treatment. (A) Example of perfectly connected QDs. (B) Dislocations in the superlattice originating from the misalignment of the $Q D$ atomic lattice. $(C)$ Example of a significantly smaller $Q D$. with a loose connection to the upper $Q D$ or the absence of connection.

It is noteworthy that the QDs can be slightly tilted toward the $\langle 110\rangle$ direction, which results in a loss of periodicity in one direction in the atomic resolution STEM image. The QDs can also be tilted from the out-of-plane direction by rotating toward the $\langle 111\rangle$ or the $\langle 110\rangle$ direction (Figures S2B and C). Figure S2D shows the misalignment of the atomic lattice between two QDs, resulting in a FFT with two orientations separated by a small angle.

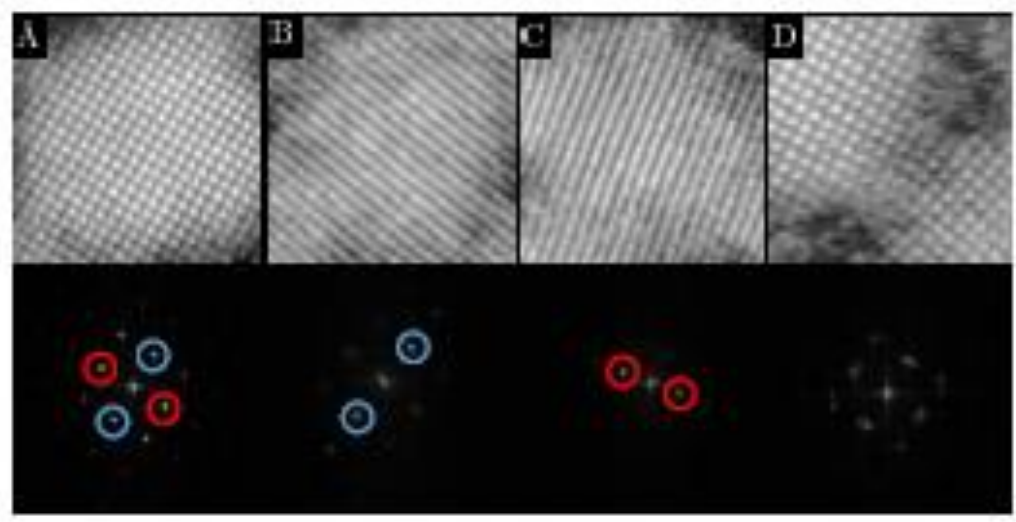

Figure S2: Disorder in the atomic lattice of QD superlattice. QDs are either aligned in the out-of-plane direction $(A)$ or tilted toward $\langle 111\rangle(B)$ or rotated toward $\langle 110\rangle(C)$. (D) Connections between two $Q D$ s where the two atomic lattices are misaligned. 
Comparison of the structural quality between as-grown and annealed superlattices of PbSe QDs
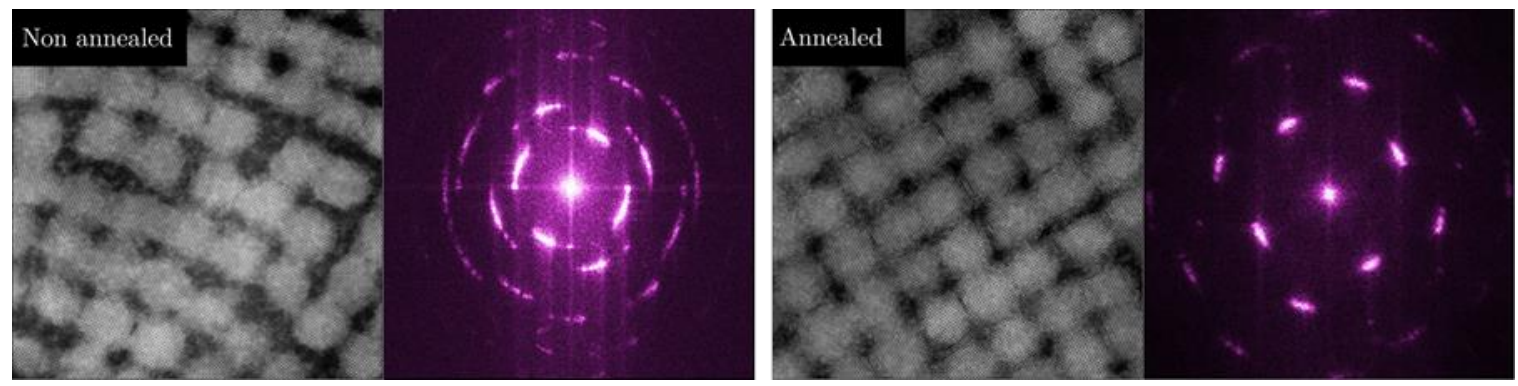

Figure S3: STEM images of a non-annealed (left) and an annealed (right) superlattices of PbSe QDS with their associated FFT, the annealing at $120{ }^{\circ} \mathrm{C}$ yielding a better alignment of the atomic planes between $Q D$ s. 


\section{Examples of differential conductance measured on individual PbSe QDs in the as-grown superlattice.}

While the spectrum shown in Figure S4(a) exhibits a classic characteristic of a single QD with discrete peaks on both sides of a ZCR, the other spectra show more diversity in their electronic features, such as the position of the Fermi level, the width of the ZCR or the peaks position. For example, the spectra c) and d) have their respective valence levels close to the Fermi level, while it is closer to the conduction levels for the spectra b) and e). Moreover, the spectra d) and e) reveal a series of peak inside the ZCR. These in-gap states most probably originate from trap states.
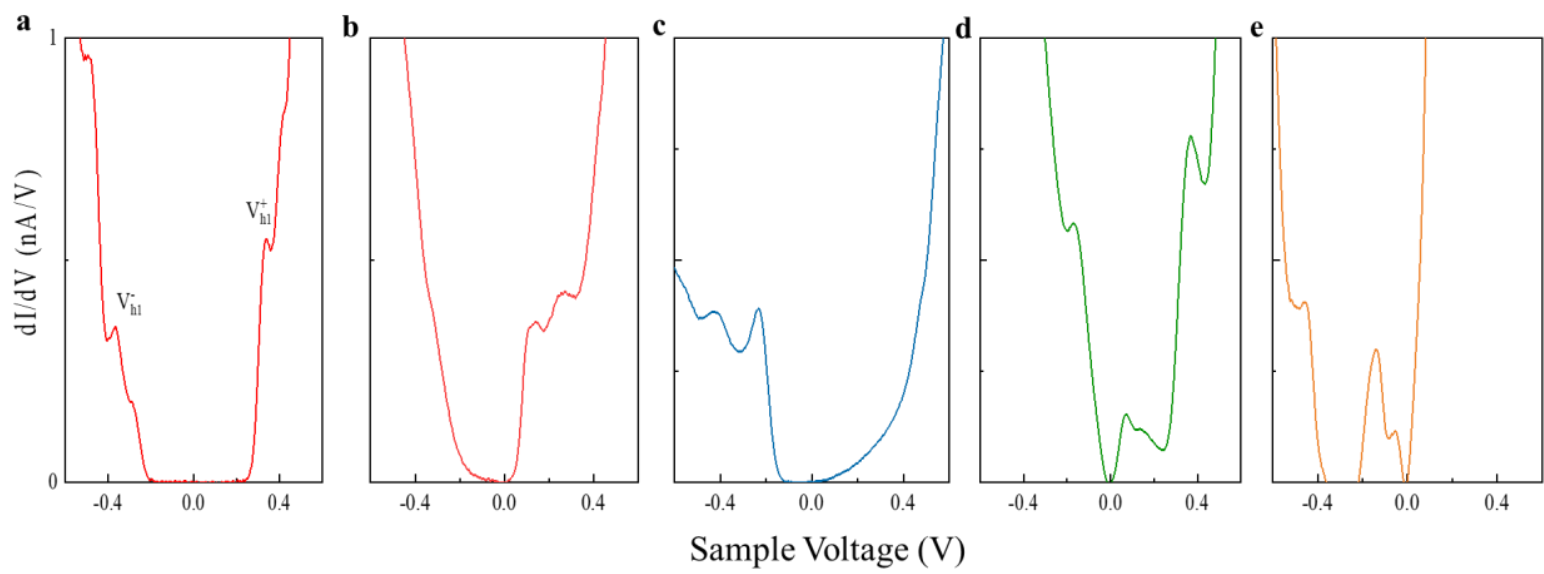

Figure S4: Differential conductance spectra acquired on five different QDs across the as-grown array and measure at a temperature of $T=77 \mathrm{~K}$. The diversity in the QDs spectra is representative of the inhomogeneous overall behavior of the sample. 


\section{Buckled QDs}

A few QDs show a much brighter contrast in the STM images of the superlattice indicating the presence of significant height variations. Figure S5a shows such an example, with the top left QDs appearing brighter than the three other QDs. A closer inspection of its height profile in comparison with the height profiles of its neighbors reveals that the brightest QD is higher by $0.9 \mathrm{~nm}$ compared to its neighbors (Figure $\mathrm{S} 5 \mathrm{~b}$ ). It is noteworthy that the size discrepancy of the QDs cannot solely account for such height variations. Indeed, the analysis of the profiles shows that the variation of the QD diameter is about 0.2-0.3 nm, much smaller than the height difference of $0.9 \mathrm{~nm}$. Moreover, this apparent size variation does not arise from the difference in the integration of the density of states to obtain the tunneling current, because the tip Fermi level is already positioned much above the top of the conduction band at a bias of $+2.5 \mathrm{~V}$. Therefore, we believe that the bright QDs reveal the presence of buckling in the superlattice. As depicted in Figure S5c, the buckling can have a tremendous importance on the electronic properties since it reduces the necking size L between the QDs, thereby hampering a strong electronic coupling.
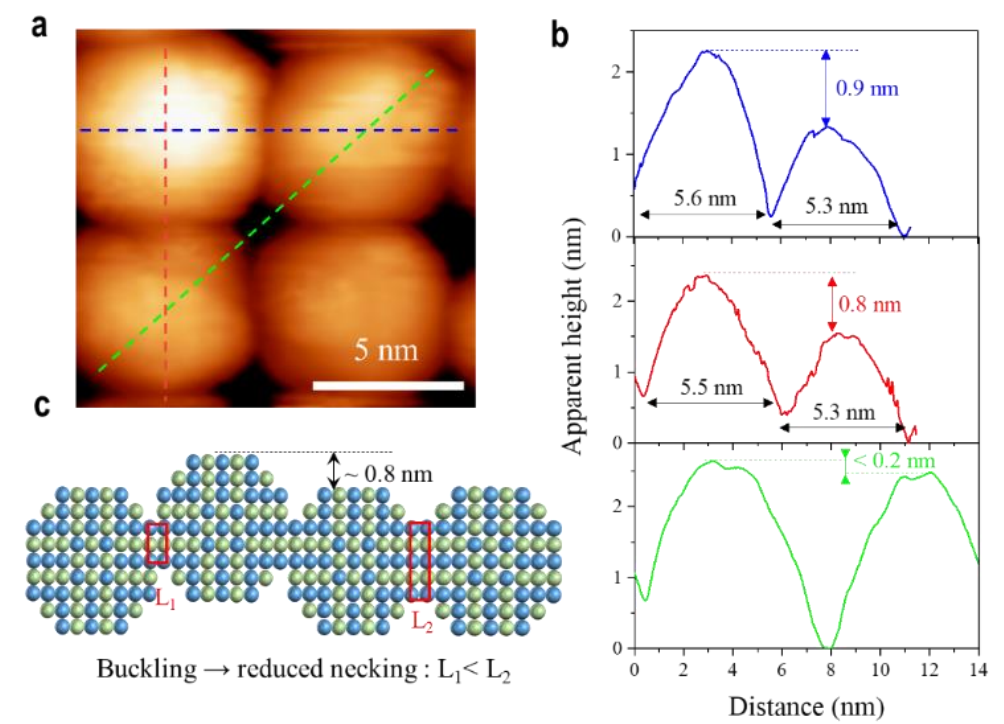

Figure S5: (a) STM image ( $\left.I_{\text {set }}=25 \mathrm{pA} ; V_{S}=+2.5 \mathrm{~V}\right)$ showing a difference in the contrast of four neighbor QDs. (b) STM height profile extracted from the lines in (a). (c) Illustration of the reduced neck width induced by the $Q D$ buckling. 
Additional examples of tunneling spectra.
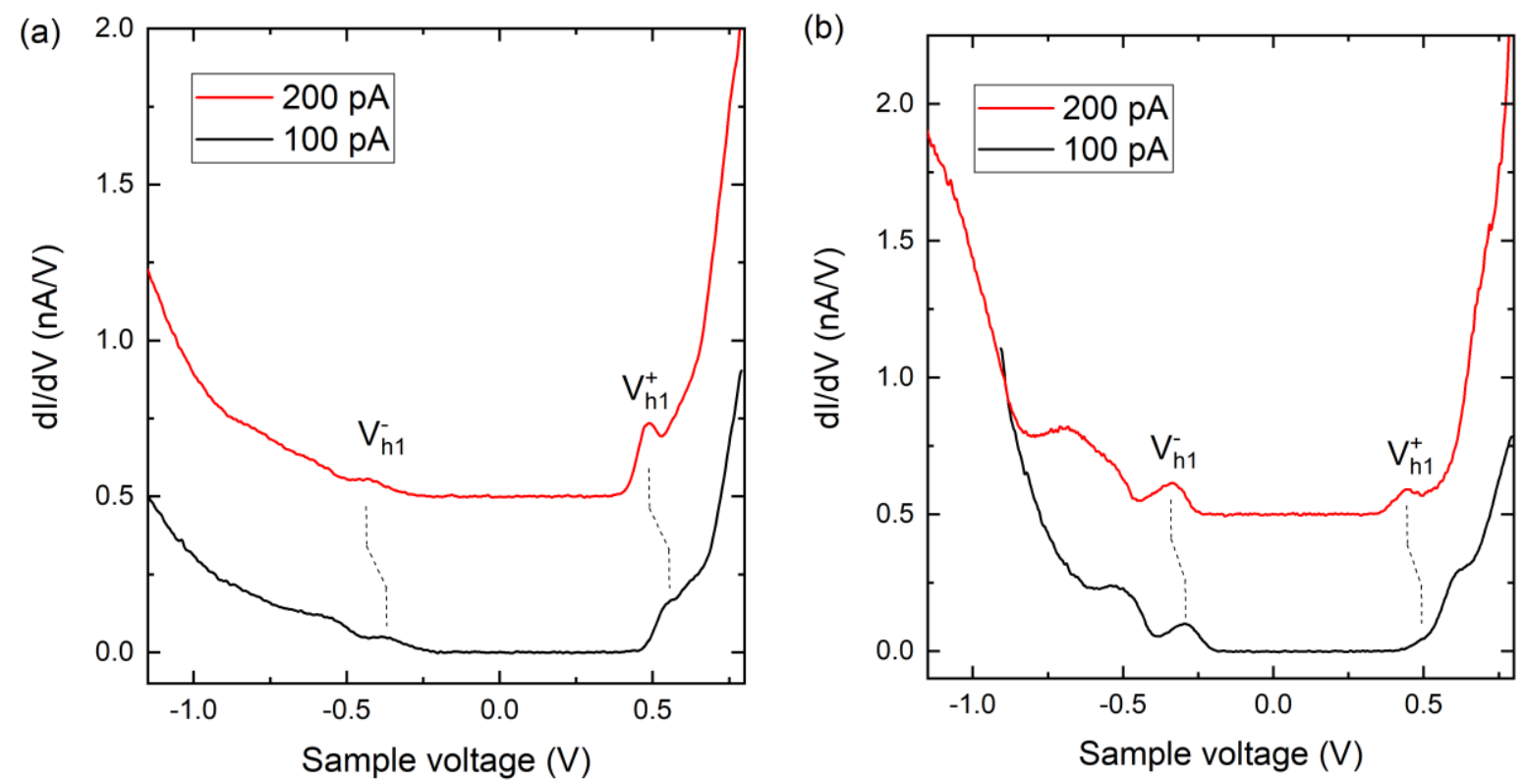

Figure S6: $d I / d V$ spectra measured on two different PbSe QDs. The use of two setpoint currents highlights, with dashed segments, the shift of the peak position towards smaller bias as the current increases. The peaks labelled $V_{h 1}^{-}$and $V^{+}{ }_{h l}$, correspond to the tunneling of holes through the ground states at negative and positive bias respectively. 


\section{Binding energy of a hydrogenic QD impurity in an epitaxially connected PbSe QD superlattice.}

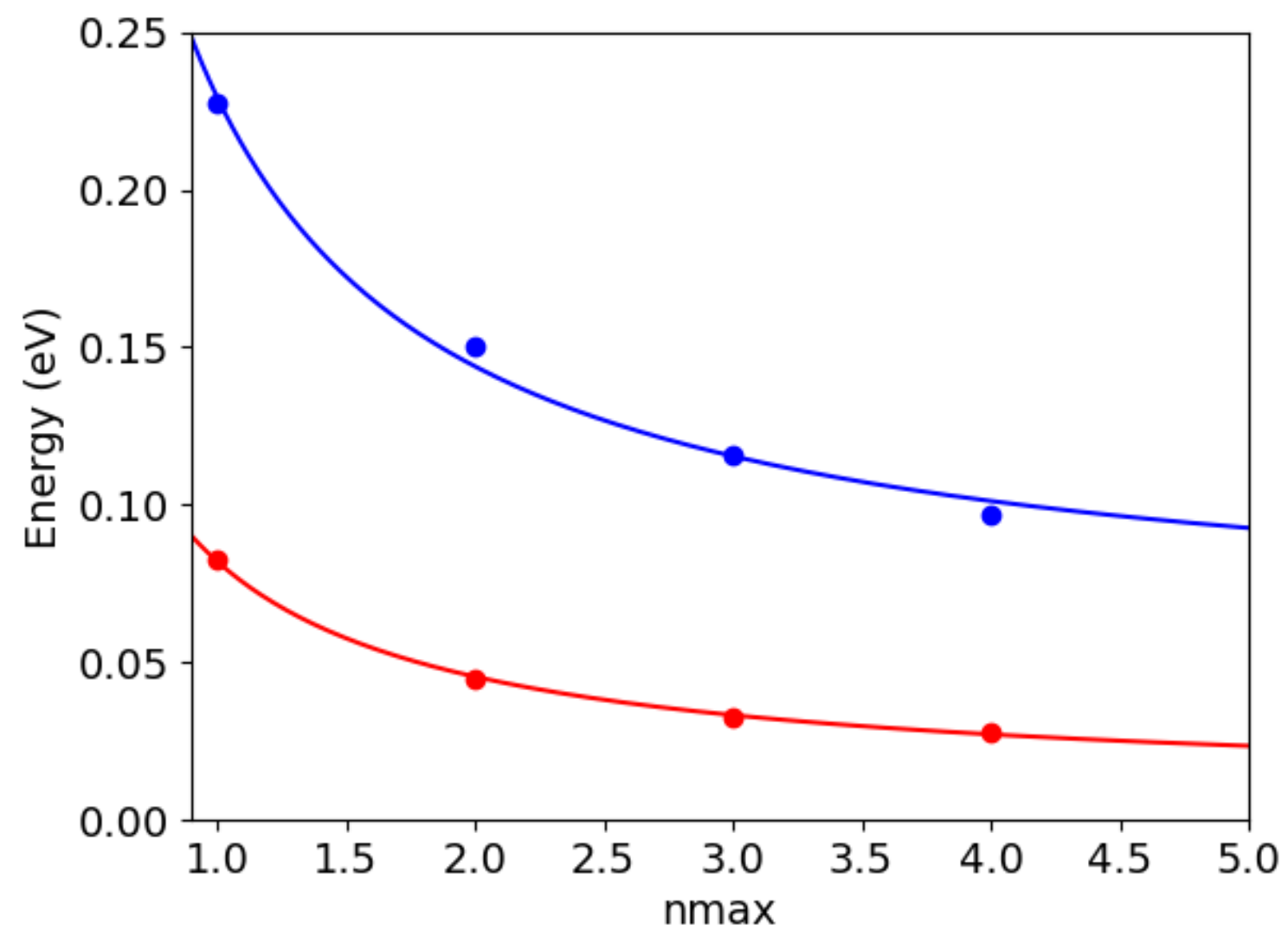

Figure S7: Binding energies of a hydrogenic QD impurity in a square array of epitaxially connected $Q D s$ with an increasing number of QDs $\left(n_{\max }\right)$. Top (blue) curve: freestanding array, bottom (red) curve: array deposited on a gold surface.

We have considered the case of an hydrogenic acceptor impurity located in a QD (values for donors are very similar). This QD is at the center of a square array of $\left(2 n_{\max }+1\right) \times\left(2 n_{\max }+\right.$ 1 ) epitaxially connected QDs. The value of $n_{\max }$ is increased in order to get the limit $n_{\max } \rightarrow$ $\infty$ by extrapolation. The hole binding energy is calculated in tight-binding in which we add the energy potential induced by the ionized impurity nuclei of charge $-e$ to the bare Hamiltonian. ${ }^{S 1}$ The screened electrostatic potential $V_{i}$ of the impurity is calculated on each atom $i$ ( $i$ varying from 1 to $N$, the number of atoms in the sample). $V_{i}$ is related to the variations of charge $\delta n_{j}$ on all atoms $j$, i.e., the induced polarization charges. As described in Ref. S2 and S3, the potentials and variations of charge are linked by a linear relation, $\delta n=\chi V$ where $\chi$ is the $N \times N$ susceptibility matrix which can be reasonably described using a bond polarizability model. The non-diagonal terms of $\chi$ are restricted to first nearest neighbors, they are all equal, and they are

(S1) Delerue, C.; Lannoo, M. Nanostructures: Theory and Modeling (Springer, 2004)

(S2) Allan, G.; Delerue, C. Energy transfer between semiconductor nanocrystals: Validity of Förster's theory. Phys. Rev. B 2007, 75, 195311.

(S3) Delerue, C.; Lannoo, M.; Allan, G. Calculations of the electron-energy-loss spectra of silicon nanostructures and porous silicon. Phys. Rev. B 1997, 56, 15306. 
adjusted to give the experimental value of the static dielectric constant in bulk PbSe. The screened potential $V$ is written as $\epsilon^{-1} V_{0}$ where $V_{0}$ is the bare (unscreened) potential of the impurity and $\epsilon$ is the dielectric matrix given by $I-v \chi$ where $I$ is the identity matrix and $v$ is the matrix of the Coulomb interaction, i.e., $v_{n, m}=e^{2} /\left|R_{n}-R_{m}\right|$ where $R_{n}$ is the position of the atom $n$. The effect of the gold substrate is taken into account by considering in the Coulomb matrix $v$ the potential of the image charges located in the metal.

The solid curves in Fig. S7 show that the hole binding energy varies as $a+b / n_{\max }$ where $a=$ $58.5 \mathrm{meV}$ and $b=170.9 \mathrm{meV}$ without the presence of the gold substrate, or $a=8.9 \mathrm{meV}$ and $b=73.2 \mathrm{meV}$ when the superlattice is in contact with the substrate. 
Table S1. Fitting parameters of the core levels shown in Figure 7.

\begin{tabular}{cccc}
\hline $\begin{array}{c}\text { Core } \\
\text { level }\end{array}$ & $\begin{array}{c}\text { Binding } \\
\text { Energy (eV) }\end{array}$ & $\begin{array}{c}\text { Spin-orbit } \\
\text { Splitting (eV) }\end{array}$ & $\begin{array}{c}\text { FWHM } \\
(\mathrm{eV})\end{array}$ \\
\hline Se 3d & 53.62 & 0.86 & 0.69 \\
$\mathrm{~Pb} \mathrm{4f}$ & 137.22 & 4.86 & 0.76 \\
& 137.91 & 4.86 & 0.76 \\
& 138.72 & 4.86 & 1.05 \\
O 1s & 529.71 & & 1.14 \\
& 531.26 & & 1.50 \\
& 532.51 & & 1.69 \\
\hline \hline
\end{tabular}

